\title{
Heuristic Contextualisation of Arithmetic Calculus by a New Network Based on the Difference Table
}

\author{
Rahman Khatibi \\ GTEV-ReX Limited: Research, Swindon, UK \\ Email: gtev.rex@gmail.com
}

How to cite this paper: Khatibi, R. (2017) Heuristic Contextualisation of Arithmetic Calculus by a New Network Based on the Difference Table. Applied Mathematics, 8, 1387-1426.

https://doi.org/10.4236/am.2017.810102

Received: September 15, 2017

Accepted: October 16, 2017

Published: October 19, 2017

Copyright $\odot 2017$ by author and Scientific Research Publishing Inc. This work is licensed under the Creative Commons Attribution International License (CC BY 4.0).

http://creativecommons.org/licenses/by/4.0/

\section{cc) (i) Open Access}

\begin{abstract}
"Arithmetic Calculus" (AC), introduced recently by the author, is explored further in this paper by giving a new lease of life to the age-old differences table by transforming it into a new kind of network. Any sequence that can be laid out in this network can be classified into one of five types of sequences, which can be expressed by algebraic polynomial or exponential functions but AC can reveal information concealed by algebra. The paper defines these sequences and refers to each family member as the parent sequence. These sequences make up their own universe as: (i) the population of the terms in each parent sequence is infinite; (ii) each parent sequence forms a hierarchy, in which their number of levels can extend into infinity; and (ii) there are infinitely diverse parent sequences. A glimpse of AC is illustrated through examples but their analytical capability is applicable in their universe. The paper shows that small sets of building blocks are operated by the convolution theorem or its variations, which is embedded "all-pervasively" in each and every member of the universe. Sufficient details are presented to ensure the emergence of the mosaic image of AC through problems including: (i) differentiation (reducement), (ii) integration (conducement), (iii) diagonal operations (reminiscent of gradient methods), and (iv) structure of hierarchies. These operations reveal that the new network can parallel Cartesian coordinates and that for problems with no noise, the deconvolution problem is well-posed against common myth of it being ill-posed.
\end{abstract}

\section{Keywords}

Arithmetic Calculus, Convolution, Conducemental Sequences, Deconvolution, Replication 


\section{Introduction}

Arithmetic Calculus (AC), introduced recently by the author [1], operates in a "universe" of number sequences, which includes those based on the power sequences $\left(x^{n}\right)$ and relates to polynomial and exponential functions. The hallmark of AC is an inbuilt analytical capability to factorise each individual member sequence, belonging to this universe, to a small set of building blocks and a set of rules operated by the convolution theorem or its variations. Each sequence in this universe is to be referred to as a parent sequence and makes up the focal point in a new hierarchical network, where AC creates a new foundational approach to mathematics and literally, no existing theorem is used in the paper but the convolutional theorem. Even this theorem is not in its traditional format but is a recast of its traditional form used in hydrology. AC is at its infancy and that by the author [1] in 2014 matches with NASA's TRL 1 (Technical Readiness Level) see [2]. This paper contextualises AC to take its status to TRL 2.

It is realised that the readership is yet to be "initiated" towards AC and therefore some initiation through [1] is assumed but Section 2 revisits certain definitions. The aims of the paper are: (i) to show that AC provides explicit formulations and reveals new information concealed by algebra; (ii) to provide further evidence that the hallmark of AC (i.e. factorisation into building blocks and convolution-based rules) is all-pervasive; (iii) to give life to a new network; and (iv) to throw a further doubt to algebraic deconvolution theorem by showing that the problem through AC is well-posed but can be ill-conditioned.

All-pervasiveness of AC is illustrated below using such basic operations as $2 \times 3$ or $(a+b+c)\left(a^{\prime}+b^{\prime}+c^{\prime}\right)$ examples as a compensation to readerships' lack of initiation.

Definition 1.1: The universe of sequences under AC uses convolution products, branded by the hallmark of: $\hat{\oplus}$; and two of its variations: referred to as regeneration products, branded by the hallmark of: $\oplus$; direct products, branded by the hallmark of: $\circ$ and $\odot$. The expressions for these products (multiplication) are reproduced in Section 2.2.

Example 1.1: Work out $2 \times 3$ using AC.

Assuming that 2 is composed of two terms 1 and 1 and also 3 is composed of three terms 1,1 , and 1 , the operation $2 \times 3$ is rewritten in the following $\mathrm{AC}$ format:

$$
\left\{\begin{array}{cc}
1.1 & 1.2 \\
\hat{1} & \hat{1}
\end{array}\right\} \hat{\oplus}\left\{\begin{array}{ccc}
2.1 & 2.2 & 2.3 \\
\hat{1} & \overrightarrow{1} & \hat{1}
\end{array}\right\}
$$

where super-fixes identify the terms with no numerical significance (i.e. $\quad \stackrel{2.2}{1}=1$ ). Thus:

$$
\left\{\begin{array}{cc}
1.1 & 1.2 \\
\hat{1} & \hat{1}
\end{array}\right\} \hat{\oplus}\left\{\begin{array}{ccc}
2.1 & 2.2 & 2.3 \\
\hat{1} & \hat{1} & \overrightarrow{1}
\end{array}\right\}
$$




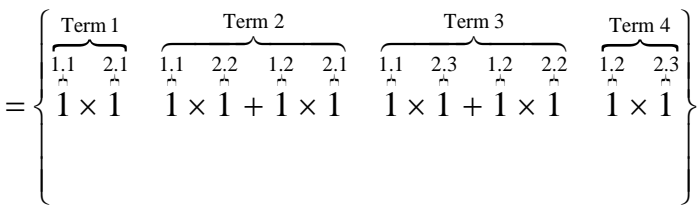

$$
\begin{aligned}
& =\left\{\begin{array}{llll}
1 & 2 & 2 & 1
\end{array}\right\}=6
\end{aligned}
$$

Remark 1.1: Note that Example 1.1 calculates $2 \times 3$ to be 6 but (i) the product is shown to be distributed as: $\left\{\begin{array}{llll}1 & 2 & 2 & 1\end{array}\right\}$ where this information is concealed in algebra; (ii) these operations are not limited to (1.1a) but " 2 " and " 3 " can be replaced with any terms, e.g. $2=0.1+0.5+0.8+0.4+0.2$, where different compositions identify different concealed information; and (iii) the procedure is general (all-pervasive) as shown in Example 1.2 below:

Example 1.2: Compare the algebraic operations for $(a+b+c)\left(a^{\prime}+b^{\prime}+c^{\prime}\right)$ with its AC counterpart.

The algebraic operations are as follows:

$$
(a+b+c)\left(a^{\prime}+b^{\prime}+c^{\prime}\right)=a a^{\prime}+a b^{\prime}+a c^{\prime}+b a^{\prime}+b b^{\prime}+b c^{\prime}+c a^{\prime}+c b^{\prime}+c c^{\prime}
$$

Although (1.2) is elementary and analytical, its inverse is not but factorised intuitively as:

$$
\begin{aligned}
& \frac{a a^{\prime}+a b^{\prime}+a c^{\prime}+b a^{\prime}+b b^{\prime}+b c^{\prime}+c a^{\prime}+c b^{\prime}+c c^{\prime}}{\left(a^{\prime}+b^{\prime}+c^{\prime}\right)} \\
& =\frac{(a+b+c)\left(a^{\prime}+b^{\prime}+c^{\prime}\right)}{\left(a^{\prime}+b^{\prime}+c^{\prime}\right)}=a+b+c
\end{aligned}
$$

The intuitive factorisation in (1.3) is not readily available in more complex operations due to the absence of an analytical approach but AC is analytical and its inverse can be handled by analytical operators. The factorised elements within each bracket are recast to their sequence formats using curly brackets and the operations are carried out, as follows:

$$
\begin{aligned}
& (a+b+c)\left(a^{\prime}+b^{\prime}+c^{\prime}\right) \equiv\left\{\begin{array}{lll}
a & b & c
\end{array}\right\} \hat{\oplus}\left\{\begin{array}{lll}
a^{\prime} & b^{\prime} & c^{\prime}
\end{array}\right\}= \\
& \left\{\begin{array}{lllll}
a & a & a & & \\
& b & b & b & \\
& & c & c & c
\end{array}\right\} \hat{\oplus}\left\{\begin{array}{lllll}
a^{\prime} & a^{\prime} & a^{\prime} & & \\
& b^{\prime} & b^{\prime} & b^{\prime} & \\
& & c^{\prime} & c^{\prime} & c^{\prime}
\end{array}\right\}= \\
& \{\overbrace{a a^{\prime}}^{\text {Term } 1} \overbrace{\left(a b^{\prime}+b a^{\prime}\right)}^{\text {Term 2 }} \overbrace{\left(a c^{\prime}+b b^{\prime}+c a^{\prime}\right)}^{\text {Term 3 }} \overbrace{\left(b c^{\prime}+c b^{\prime}\right)}^{\text {Term 4 }}{\stackrel{\overbrace{}}{c c^{\prime}}}_{\text {Term }}^{5}\} \\
& \equiv \overbrace{a a^{\prime}}^{\text {Term } 1}+\overbrace{\left(a b^{\prime}+b a^{\prime}\right)}^{\text {Term } 2}+\overbrace{\left(a c^{\prime}+b b^{\prime}+c a^{\prime}\right)}^{\text {Term } 3}+\overbrace{\left(b c^{\prime}+c b^{\prime}\right)}^{\text {Term } 4}+\overbrace{c c^{\prime}}^{\text {Term } 5}
\end{aligned}
$$

Algebra is not minded with the inverse of $(a+b+c)$ or $\left(a^{\prime}+b^{\prime}+c^{\prime}\right)$; whereas the inverse of any AC quantities is conjugated with their direct formats and in this way AC is capable of handling its inverse through analytical procedures. The direct format is expressed by $(1.4 \mathrm{~d})$ and its inverse is presented through the example below: 
Example 1.3: Carry out the inverse of (1.4d) and divide the product by: $\left\{\begin{array}{lll}a & b & c\end{array}\right\}$.

First step: work out the inverse of $\left\{\begin{array}{lll}a & b & c\end{array}\right\}$ and by definition its product with its inverse must be unity, expressed as follows:

$$
\left\{\begin{array}{lll}
a & b & c
\end{array}\right\} \hat{\oplus}\left\{\begin{array}{llllll}
p & q & r & s & t & \cdots
\end{array}\right\}=\left\{\begin{array}{llllll}
1 & 0 & 0 & 0 & 0 & \cdots
\end{array}\right\}
$$

Second step: Form five equations from (1.5a) and solve the 5 unknowns ( $p, q$, $r, s, t)$ :

$$
\begin{aligned}
& \text { (i) } p=\frac{1}{a} ; \quad \text { (ii) } q=-\frac{b}{a^{2}} \text {; (iii) } r=\frac{b^{2}-a c}{a^{3}} ; \text { (iv) } s=\frac{2 a b c-b^{3}}{a^{4}} ; \\
& t=\frac{b^{4}-3 a b^{2} c+a^{2} c^{2}}{a^{5}}: \\
& \therefore \frac{1}{\left\{a^{\prime} b^{\prime} c^{\prime}\right\}}=\hat{\oplus}\left\{\frac{1}{a}-\frac{b}{a^{2}} \frac{b^{2}-a c}{a^{3}} \frac{2 a b c-b^{3}}{a^{4}} \frac{b^{4}-3 a b^{2} c+a^{2} c^{2}}{a^{5}}\right\}
\end{aligned}
$$

Third step: Express the AC analytical inverse operations, which are as follows:

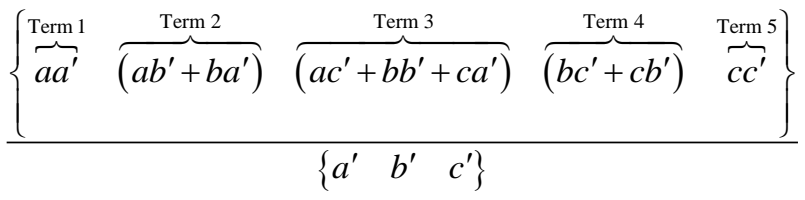

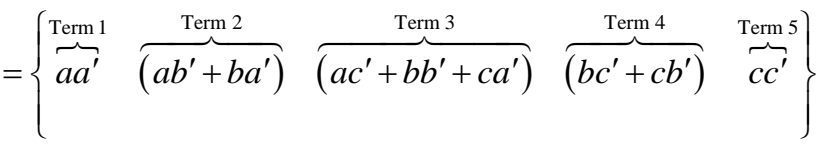

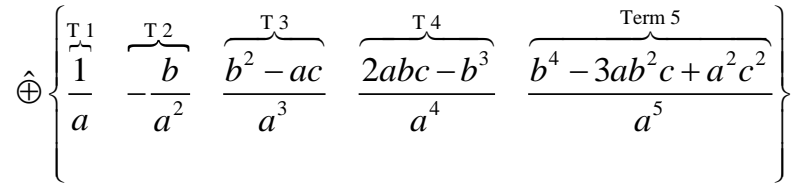

$$
\begin{aligned}
& =\left\{\begin{array}{lllll}
a^{\prime} & b^{\prime} & c^{\prime} & 0 & 0
\end{array}\right\}
\end{aligned}
$$

The operations in (1.6) simply do not exist in algebra, but they are AC constructs through the convolution theorem. To compare AC with algebra, a further example is given, which uses the elements of the Pascal Triangle for Examples 1.2 and 1.3, as follows:

Example 1.4: Consider the Pascal Triangle for the case of $\left\{\begin{array}{lll}1 & 2 & 1\end{array}\right\}$ to produce: $\left\{\begin{array}{lllll}1 & 4 & 6 & 4 & 1\end{array}\right\}$.

There are few algebraic ways to carry out the above operations but AC operations as per (1.4) for $a=a^{\prime}=1, b=b^{\prime}=2$ and $c=c^{\prime}=1$ are as follows:

$$
\left\{\begin{array}{lll}
1 & 2 & 1
\end{array}\right\} \hat{\oplus}\left\{\begin{array}{lll}
1 & 2 & 1
\end{array}\right\}=\left\{\begin{array}{lllll}
1 & 4 & 6 & 4 & 1
\end{array}\right\}
$$

The inverse operation of (1.7) is equivalent to the case of $\left\{\begin{array}{lllll}1 & 4 & 6 & 4 & 1\end{array}\right\}$ divided by $\left\{\begin{array}{lll}1 & 2 & 1\end{array}\right\}$. It is widely known that the inverse of $\left\{\begin{array}{lll}1 & 2 & 1\end{array}\right\}$ is given by:

$$
\frac{1}{\left\{\begin{array}{lll}
1 & 2 & 1
\end{array}\right\}}=\left\{\begin{array}{lllll}
1 & -2 & 3 & -4 & 5
\end{array}\right\}
$$

Algebra lacks an operator for the product of these two but AC is operated as 
follows:

$$
\begin{aligned}
& \left\{\begin{array}{lllll}
1 & 4 & 6 & 4 & 1
\end{array}\right\} \oplus\left\{\begin{array}{lllll}
1 & -2 & 3 & -4 & 5
\end{array}\right\} \\
& =\left\{\begin{array}{llll}
1 \times 1 & (-1 \times 2+4 \times 1) & (1 \times 3-4 \times 2+6 \times 1) & (-1 \times 4+4 \times 3-6 \times 2+4 \times 1)
\end{array}\right. \\
& (1 \times 5-4 \times 4+6 \times 3-4 \times 2+1 \times 1)\} \\
& =\left\{\begin{array}{lllll}
1 & 2 & 1 & 0 & 0
\end{array}\right\}
\end{aligned}
$$

The above examples illustrate a major difference between traditional algebra and AC, according to which, algebra is a set of clever operations and when it has problems, it either remains as low as intuition or becomes very sophisticated, e.g. the deconvolution theorem. On the other hand, AC needs initiation; its operations are logical but not apparent and this explains its belated discovery; it creates a procedural approach even for its inverse problems; thus, AC is smart. Whilst the above examples are given by everyday algebraic experience, the remaining sections focus on sequences with higher complexity.

\section{Initiation of AC-Prerequisite Background}

\subsection{Basic Definitions}

AC is associated with a set of proposed terms, new symbols and new operators, presented by the author [1], which may be referenced for full details but basic definitions are reproduced here for continuity and warnings are given where the definitions have been revised.

Definition 2.1-sequences: A sequence of numbers is a collection of highly ordered set of individual numbers, each referred to as a term, or an element, separated by a comma or space and denoted by a curly bracket, e.g.:

$$
\{\mathbb{N}\}=\left\{\begin{array}{lllllll}
1 & 2 & 3 & \cdots & i & \cdots & n
\end{array}\right\}
$$

Definition 2.2-Bounded/ unbounded sequences. The number of the terms in bounded sequences is finite but in unbounded sequences, it is not. Bounded sequences are denoted by upper case single strike letters: $\{\mathcal{S}\}$ but unbounded sequences by upper cases double strike letters: $\{\mathbb{S}\}$ and natural numbers are denoted by $\{\mathbb{N}\}$.

Definition 2.3-Regeneration: This is a way of using a sequence at a lower level and building a new sequence at a higher level by operating on its terms through summing, where the process of regeneration for each term starts from the first term, see operators (2.6).

Definition 2.4-Arithmetic Calculus ( $A C$ ): is a proposed term to refer to a new branch of mathematics, in which sequences are likened to assets and the theory of convolution or its variations are likened to currency. AC covers a universe of sequences of infinite sizes. These sequences are to be referred to as conducemental sequences, where the justification for coining this term is given by [1].

Definition 2.5-Conducemental Sequence: Any sequences involving a multiplication by the convolution theorem or its variations are to be referred to as 
conducemental sequences, e.g. the Pascal Triangle and the sequence of natural numbers.

Definition 2.6: Conducemental Sequences refer to a whole universe of synthetic sequences based on (i) a kernel, e.g. $\langle 1\rangle$ or $\left\langle\begin{array}{ll}1 & 1\rangle\end{array}\right.$; (ii) a sequence of counters, which may be unbounded, e.g.: $\left\{\begin{array}{lllll}1 & 1 & 1 & 1 & \ldots\end{array}\right\}$, or bounded, e.g. $\left\{\begin{array}{llll}1 & 1 & 1 & 1\end{array}\right\}$; (iii) multiplication of these constituent sequences by employing one or more of the product rules (defined in Section 2.2); (iv) the source term (first term) of the sequence may start from any term; and (v) some of the terms may be defective.

Definition 2.7-conducemental dimension specifications. The focus of attention in conducemental sequences is on parent sequences, $\{\mathbb{S}\}$, which is normally associated with another one to be referred to base sequences, $\left\{\mathbb{S}^{\prime}\right\}$. In between the parent and the base sequences, as well as outside them, there are many sequences at different hierarchies but all of them can be specified as:

$\{\mathcal{D S} \mid \mathbb{S}\}=\left[\left\langle\mathcal{A S} \mid d\left(d_{f}, d_{t}\right), \sigma, g\right\rangle\left\langle\left\langle\mathcal{L S} \mid \sigma_{s}\right\rangle \quad\right.\right.$ Defect $\left.\left\langle\mathcal{K} \mid \mathcal{T}_{1} \mathcal{T}_{2} \quad \cdots \quad \mathcal{T}_{\sigma}\right\rangle\right](2.2 \mathrm{a})$

which is read as dimensional specification of $\mathbb{S}$ is given by i) architectural specification in terms of its degree from ... to ..., order and generation; ii) logistic specification in terms of its ordinal of the source term; iii) any defect in any of the term; and iv) specification of it kernel. The specification in (2.2a) comprises the following four components:

i) Architecture Specification $(\mathcal{A S})$ : This comprises: degree: $d$ (degree often involves operations in a range from one degree, $d_{f}$, to another degree, $d_{t}$ ); order. $\sigma$, and generation: $g$. The specification using the kernels is denoted by $\delta$ and $\omega$.

ii) Logistics Specification $(\mathcal{L S})$ : It comprises ordinal of the source terms: $\emptyset_{s}$.

iii) Defect/mutation Specification: It specifies the term(s) suffering from errors.

iv) Kernel Specification $\mathcal{K}$ : It comprises size (and this is the same as order), values of its individual terms and the sum of these individual values.

Remark 2.1: The propositions "from" and "to" are underlined for emphasis.

Warning: Definitions for order and degree in the paper are opposites of those in [1].

Example 2.1: Consider the following examples to show their specifications:

For $\{\mathbb{N}\}=\left\{\begin{array}{llll}1 & 2 & \cdots & n\end{array}\right\}=\left\{\left(\begin{array}{l}1 \\ 0\end{array}\right)\left(\begin{array}{l}2 \\ 1\end{array}\right) \cdots\left(\begin{array}{c}n \\ n-1\end{array}\right)\right\}$

Specification is:

$$
\lceil\langle\mathcal{A S} \mid 1,1,2\rangle\langle\mathcal{L S} \mid 1\rangle \quad \text { Defect } \mathcal{K} \mid\langle 1\rangle\rceil
$$

For $\{\mathbb{S}\}=\left\{\begin{array}{llll}1^{3} & 2^{3} & \cdots & n^{3}\end{array}\right\}=\left\{\left\{\begin{array}{lll}1 & 4 & 1\end{array}\right\} \odot\left\{\left(\begin{array}{c}n+2 \\ n-1\end{array}\right)\left(\begin{array}{c}n+1 \\ n-2\end{array}\right)\left(\begin{array}{c}n \\ n-3\end{array}\right)\right\}\right\}$

Specification is:

$$
\left\lceil\langle\mathcal{A S} \mid 3,3,0\rangle\langle\mathcal{L S} \mid 1\rangle \quad \text { Defect } \mathcal{K} \mid\left\langle\begin{array}{lll}
1 & 4 & 1\rangle
\end{array}\right]\right.
$$




$$
\text { For }\{\mathbb{S}\}=\left\{\begin{array}{llll}
1^{2} & 3^{2} & \cdots & (2 n-1)^{2}
\end{array}\right\}=\left\{\begin{array}{lll}
1 & 6 & 1
\end{array}\right\} \odot\left\{\left(\begin{array}{l}
n+1 \\
n-1
\end{array}\right)\left(\begin{array}{c}
n \\
n-2
\end{array}\right)\left(\begin{array}{c}
n-1 \\
n-3
\end{array}\right)\right\}
$$

Specification is:

$$
\left\lceil\langle\mathcal{A S} \mid 4,3,0\rangle\langle\mathcal{L S} \mid 1\rangle \text { Defect } \quad \mathcal{K} \mid\left\langle\begin{array}{lll}
1 & 6 & 1\rangle
\end{array}\right.\right.
$$

Definition 2.8-Reducement and Conducement. Two fundamental operations of differentiation and integration are referred to in $\mathrm{AC}$ by the proposed terms of reducement and conducement. The inverse of reducement is conducement. These proposed terms are not standard and they should go with the propositions: From one hierarchy ( $d_{f}$ - degree from) to another ( $d_{t}$ degree to).

Conducemental sequences have the hierarchy of: (i) a parent sequence with their terms ranging from $-\infty$ to $\infty$ sequentially (horizontally, along rows, or ordinal-wise) and hierarchically (vertically, or degree-wise). The paper aims to capture the inherent sense of interconnection and interrelation within this network with the potential to infinity-within-infinity. This network sense is ignored in algebra but is brought to life by AC.

Remark 2.2: Hybrid terms have also been made from these two proposed terms, e.g. conducemental algebra, exemplified by the operations in Examples 1.1 - 1.4. Also, the paper uses two derivative terms of conducemental and reducemental to express the products of building blocks and rules for typical single term. Conducemental sequences are classified and various types are associated with proposed terms, as to be given in due course.

Remark 2.3: is used to show that this parameter is not considerd.

\subsection{New Operators}

Multiplication rules, presented below, operate on the corresponding elements of two sequences, as follows:

$$
\begin{aligned}
& \left\{\begin{array}{lllll}
t_{1} & t_{2} & \cdots & t_{n-1} & t_{n}
\end{array}\right\} \\
& \left\{\begin{array}{lllll}
r_{1} & r_{2} & \cdots & r_{m-1} & r_{m}
\end{array}\right\}
\end{aligned}
$$

where $m$ and $n$ can be any large value. Both (2.3a) and (2.3b) can be sets of numbers or sequences. One of them acts as rules and the other as building blocks or invariants. The following multiplication rules are presented.

\subsubsection{Direct Products}

The direct product rule (or dot products) is defined as:

$$
\begin{gathered}
\left\{\begin{array}{lllll}
t_{1} & t_{2} & \cdots & t_{n-1} & t_{n}
\end{array}\right\} \circ\left\{\begin{array}{lllll}
r_{1} & r_{2} & \cdots & r_{n-1} & r_{n}
\end{array}\right\} \\
=\left\{\begin{array}{llllll}
t_{1} r_{1} & t_{2} r_{2} & t_{3} r_{3} & \cdots & t_{n-1} r_{n-1} & \left.t_{n} r_{n}\right\}
\end{array}\right. \\
\left\{\begin{array}{llllll}
t_{1} & t_{2} & t_{3} & \cdots
\end{array}\right\} \odot\left\{\begin{array}{lllll}
r_{1} & r_{2} & r_{3} & \cdots
\end{array}\right\}=\left\{\begin{array}{llll}
t_{1} r_{1}+t_{2} r_{2}+t_{3} r_{3} & \cdots & t_{n} r_{n}
\end{array}\right\}
\end{gathered}
$$

In (2.4a), dot products are denoted by “。” but the summing from the first term in $(2.4 \mathrm{~b})$ is denoted by “ $\odot . "$

Example 2.2: The examples below exemplify the direct product rule: 


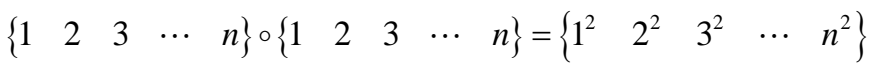

$$
\begin{aligned}
& \left\{\begin{array}{lllll}
1 & 2 & 3 & \cdots & n
\end{array}\right\} \odot\left\{\begin{array}{lllll}
1 & 2 & 3 & \cdots & n
\end{array}\right\}=\left\{1^{2}+2^{2}+\cdots+n^{2}\right\}
\end{aligned}
$$

\subsubsection{Regeneration Products}

The regenerating product rule is defined below:

$$
\begin{aligned}
\left\{\begin{array}{lllll}
t_{1} & t_{2} & \cdots & t_{n-1} & t_{n}
\end{array}\right\}\left\{\begin{array}{lllll}
r_{1} & r_{2} & \cdots & r_{n-1} & r_{n}
\end{array}\right\} \\
=\left\{\begin{array}{lllll}
t_{1} r_{1} & t_{1} r_{2}+t_{2} r_{1} & t_{1} r_{3}+t_{2} r_{2}+t_{3} r_{1} & \cdots & t_{1} r_{m-1}+t_{2} r_{m-2}+\cdots+t_{n-2} r_{2}+t_{n-1} r_{1}
\end{array}\right. \\
\left.t_{1} r_{m}+t_{2} r_{m-1}+\cdots+t_{n-1} r_{2}+t_{n} r_{1}\right\}
\end{aligned}
$$

Remark 2.4: Both (2.5) and (2.6) have recursive versions but are not used in the paper.

The number of terms in the product is equal to the largest value of $m$ or $n$. As shown in Appendix I, the above format is a direct adoption from the convolution theorem used in hydrology. The convolution theorem has been applied widely and goes back to Jean-Baptiste le Rond d'Alembert (1717-1783) and Pierre-Simon, marquis de Laplace (1749-1827) and others, see [3] for details but surprisingly it has not penetrated to generating functions as detailed in [4].

\subsubsection{Convolution Products}

The convoluting product rule is defined as:

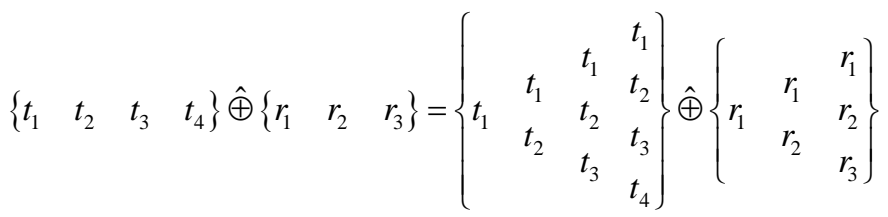

$$
\begin{aligned}
& =\left\{\begin{array}{llllll}
t_{1} r_{1} & t_{1} r_{2}+t_{2} r_{1} & t_{1} r_{3}+t_{2} r_{2}+t_{3} r_{1} & t_{2} r_{3}+t_{3} r_{2}+t_{4} r_{1} & t_{3} r_{2}+t_{4} r_{1} & t_{4} r_{1}
\end{array}\right\}
\end{aligned}
$$

The last two terms: $t_{3} r_{2}+t_{4} r_{1}$ and $t_{4} r_{1}$, cause dipping in the trailing values of the product, as may be seen in the example below.

Example 2.3: Use the terms of Pascal Triangle to show the convolution product rule:

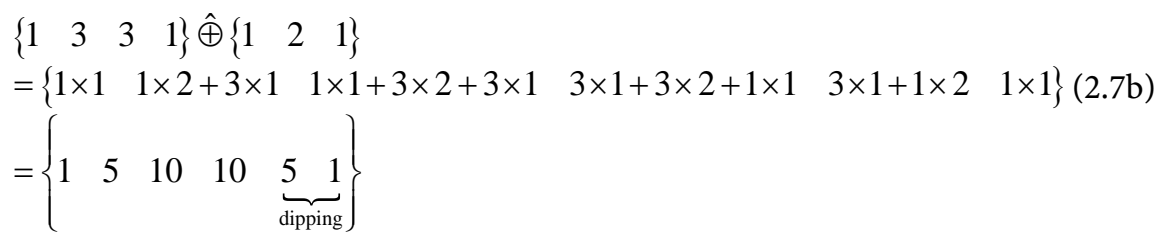

Hence, the over-fix in the notation, $\hat{\oplus}$, reflects the dipping at the trailing elements.

\subsection{New Basic Sequences}

The AC capability is underpinned by a host of basic conducemental sequences, which are captured in Table 1 in mathematical terms, including their constituent sequence used for their conducemental/reducementals. This gives a unified labelling scope in natural language (uninomial, binomial, trinomial, quadrinomial and polynomial). 
Table 1. Uninomial, binomial and trinomial sequences built on natural numbers (Remark 2.5: $\mathbb{B}_{1}$ of ID 6 in the table is arithmetic progression).

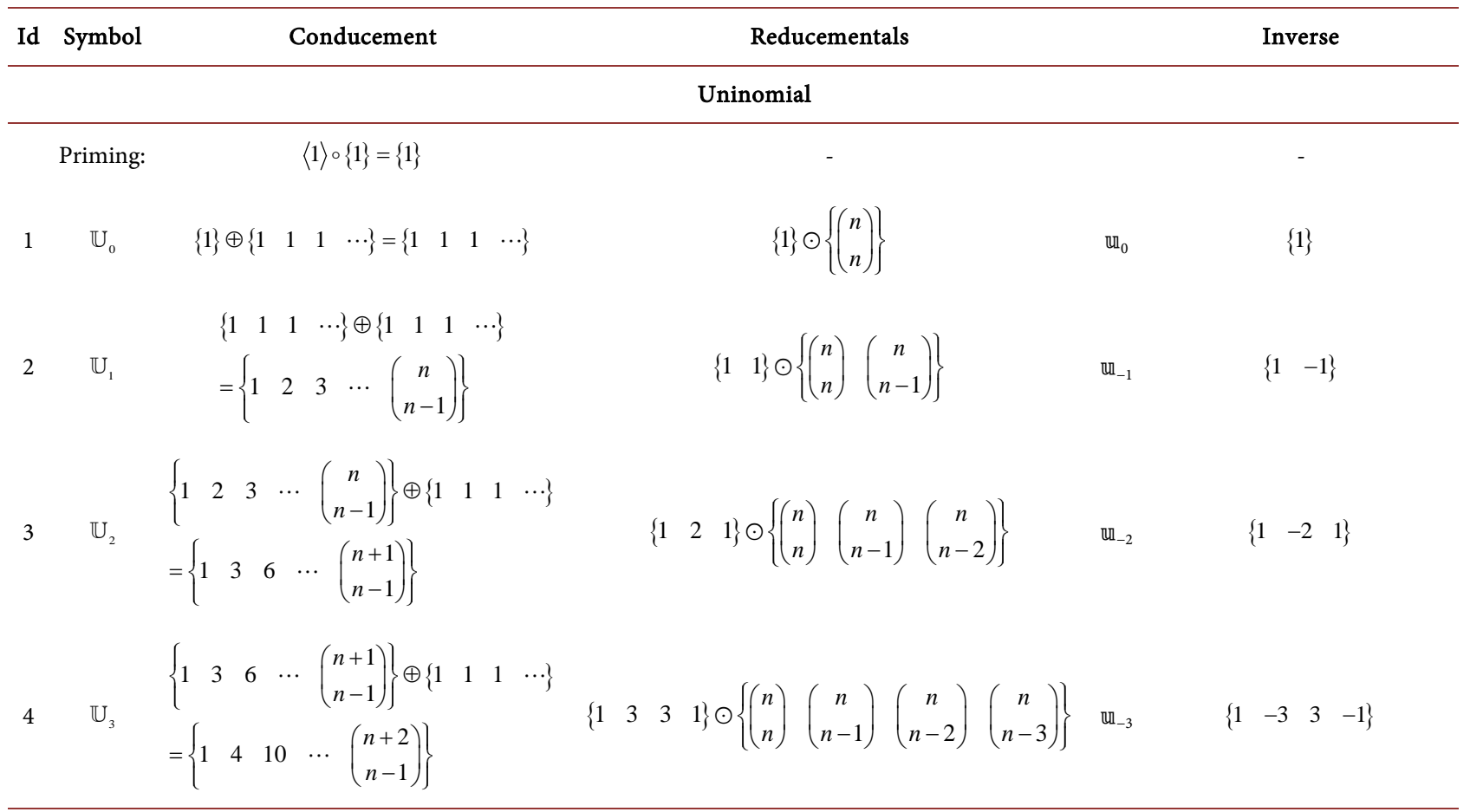

\section{Binomial}

5 Priming: $\quad\left\langle\begin{array}{ll}1 & 1\end{array}\right\rangle \circ\left\{\begin{array}{ll}1 & 1\end{array}\right\}=\left\{\begin{array}{ll}1 & 1\end{array}\right\}$

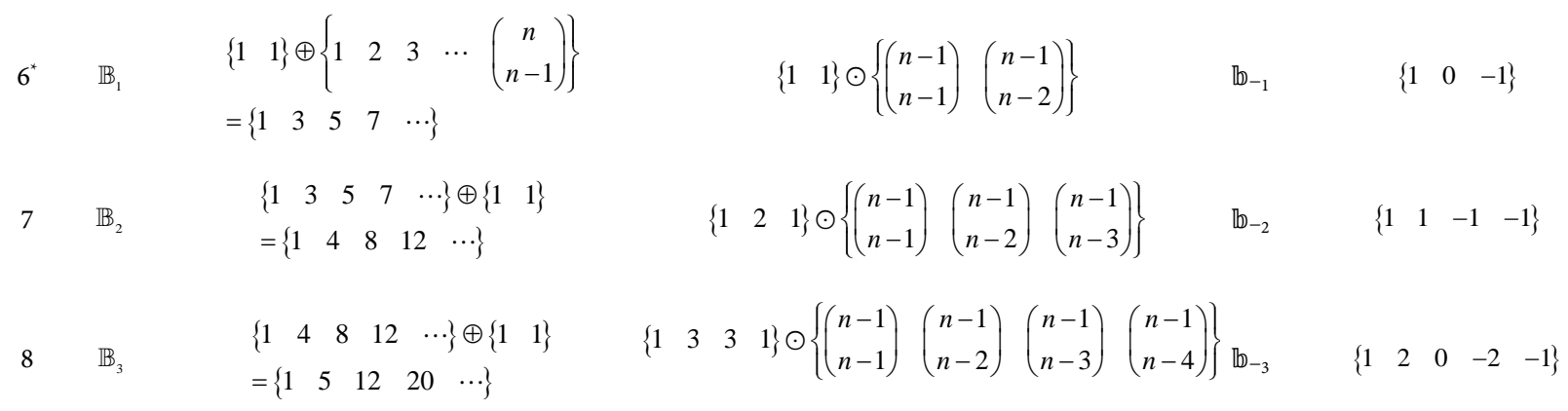

Trinomial Sequences

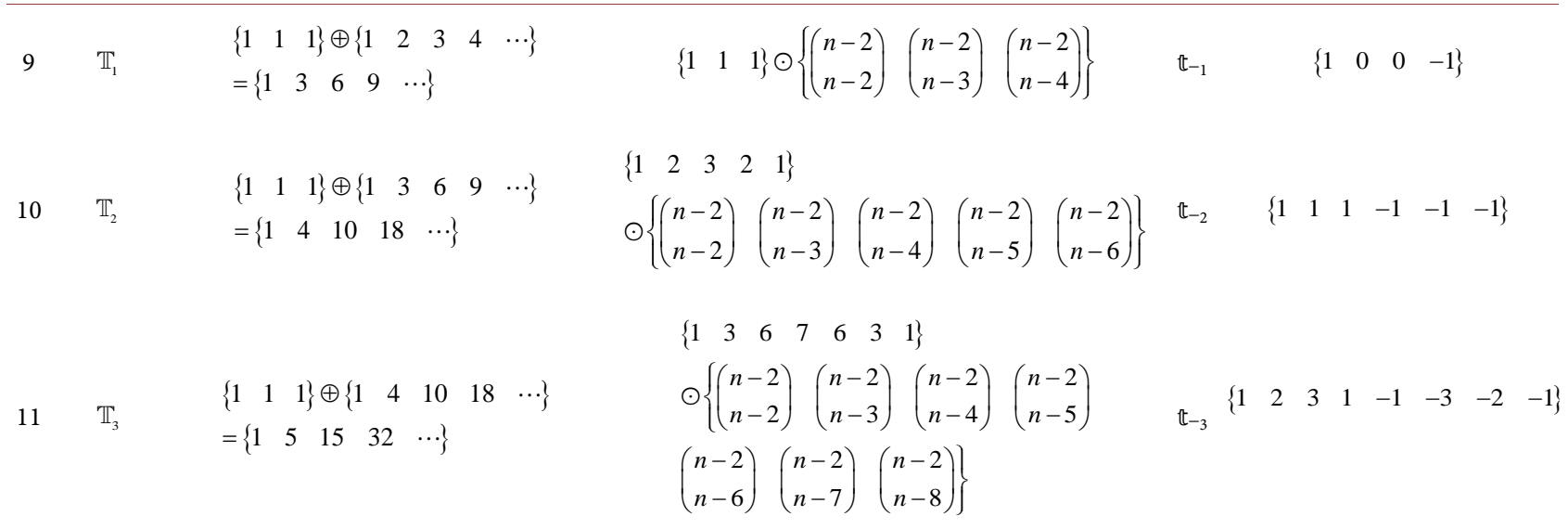


The proposed shorthand mathematical symbols for denoting the above unbounded and bounded conducemental sequence forms are as follows:

- For unbounded conducemental sequences, use: $\mathbb{U}, \mathbb{B}, \mathbb{T}, \mathbb{Q}, \mathbb{P}$;

- For and their inverses, use lower-case double-strike scripts: ㄸ, $\mathbb{b}, \mathfrak{t}, \mathbb{q}, \mathbb{P}$

- e.g. $\left\{\mathbb{U}_{0}\right\}=\left\{\begin{array}{lllll}1 & 1 & 1 & 1 & \cdots\end{array}\right\}$ and $\left\{\mathbb{u}_{-1}\right\}=\left\{\begin{array}{ll}1 & -1\end{array}\right\}$

- For bounded conducemental use upper-case single-strike scripts e.g. $\left\{\mathcal{B}_{2}\right\}=\left\{\begin{array}{lll}1 & 2 & 1\end{array}\right\}$. For their inverses, use their lowercase scripts, e.g. $\left\{b_{2}\right\}=$ $\left\{\begin{array}{llll}1 & -2 & 3 & \cdots\end{array}\right\}$.

Uninomial sequences in AC are pivotal, which are cut-and-shaped through the convolution theorem and its variations to make the bulk of AC. The pivotal role of the uninomial sequences cannot be overemphasised.

\subsection{Combination of Negative Numbers and Conducemental Table}

Combinatorial operators with negative numbers are treated as follows:

Definition 2.9: The following is well-established in mathematics:

Remark 2.6: Sequence of counters for reducementals are all binomial expressed: $\left\{\left(\begin{array}{l}n-2 \\ n-2\end{array}\right)\left(\begin{array}{l}n-2 \\ n-3\end{array}\right)\left(\begin{array}{l}n-2 \\ n-4\end{array}\right)\left(\begin{array}{l}n-2 \\ n-5\end{array}\right)\left(\begin{array}{l}n-2 \\ n-6\end{array}\right) \quad \ldots\right\}$ Consider it for $n$ $=6$, which render the sequence: $\left\{\begin{array}{lllllll}0 & 0 & 1 & 4 & 6 & 4 & 1\end{array}\right\}$ and for $n=7$, it renders:: $\left\{\begin{array}{lllllllll}0 & 0 & 0 & 1 & 5 & 10 & 10 & 5 & 1\end{array}\right\}$ and therefore this is a convenient way of shifting the source term to higher ordinals.

$$
\left(\begin{array}{c}
-(n) \\
i
\end{array}\right)=\left\{(-1)^{n}\left(\begin{array}{c}
n \\
i
\end{array}\right)\right\}
$$

However, negative base values in combinatorics operation is not allowed.

Definition 2.10: extension to negative numbers: Currently the operator for choosing $m$ objects from a set of $n$ objects applies to positive numbers but its application to negative numbers runs to problems. The following transformation removes the problem:

$$
\left(\begin{array}{c}
n+i \\
n-j
\end{array}\right) \Rightarrow \frac{n}{|n|}\left(\begin{array}{c}
|n|+i \\
|n|-j
\end{array}\right)
$$

where $n$ is variable and can be negative, $i$ and $j$ values are positive and integer. The table of difference or Conducemental Table is generalised and is given in Table 2.

\section{Arithmetic Calculus in a Nutshell}

Arithmetic calculus turns attention away from the sequence as a whole but focuses on the individual terms and each reducemental is an AC expression for the individual terms. An account below shows the way for this transformation.

\subsection{Transforming Difference Tables into a New Network}

The difference table is well known in mathematics but its origin is obscure, although it was known to Chinese astronomers in the $8^{\text {th }}$ century [5]. It also re- 
Table 2. Extended conducemental table for uninomial sequences.

\begin{tabular}{|c|c|c|c|c|c|c|c|c|c|c|c|c|c|c|c|c|}
\hline Gen. & Deg & $-\mathrm{g} 6$ & $-\mathrm{g} 5$ & $-\mathrm{g} 4$ & $-g 3$ & $-\mathrm{g} 2$ & $-\mathrm{g} 1$ & g0 & g1 & g2 & g3 & g4 & g5 & g6 & Deg & Gen \\
\hline$-\mathrm{g} 10$ & $\mathbb{U}_{-9}$ & -2002 & -715 & -220 & -55 & -10 & -1 & 0 & 1 & 10 & 55 & 220 & 715 & 2002 & $\mathbb{U}_{9}$ & g10 \\
\hline$-\mathrm{g} 9$ & $\mathbb{U}_{-8}$ & -1287 & -495 & -165 & -45 & -9 & -1 & 0 & 1 & 9 & 45 & 165 & 495 & 1287 & $\mathbb{U}_{8}$ & g9 \\
\hline$-g 8$ & $\mathbb{U}_{-7}$ & -792 & -330 & -120 & -36 & -8 & -1 & 0 & 1 & 8 & 36 & 120 & 330 & 792 & $\mathbb{U}_{7}$ & g8 \\
\hline$-\mathrm{g} 7$ & $\mathbb{U}_{-6}$ & -462 & -210 & -84 & -28 & -7 & -1 & 0 & 1 & 7 & 28 & 84 & 210 & 462 & $\mathbb{U}_{6}$ & g7 \\
\hline$-g 6$ & $\mathbb{U}_{-5}$ & -252 & -126 & -56 & -21 & -6 & -1 & 0 & 1 & 6 & 21 & 56 & 126 & 252 & $\mathbb{U}_{5}$ & g6 \\
\hline$-g 5$ & $\mathbb{U}_{-4}$ & -126 & -70 & -35 & -15 & -5 & -1 & 0 & & 5 & 15 & 35 & 70 & 126 & $\mathbb{U}_{4}$ & g5 \\
\hline$-\mathrm{g} 4$ & $\mathbb{U}_{-3}$ & -56 & -35 & -20 & -10 & -4 & -1 & 0 & & & 10 & 20 & 35 & 56 & $\mathbb{U}_{3}$ & $\mathrm{~g} 4$ \\
\hline$-\mathrm{g} 3$ & $\mathbb{U}_{-2}$ & -21 & -15 & -10 & -6 & -3 & -1 & 0 & & & & 10 & 15 & 21 & $\mathbb{U}_{2}$ & g3 \\
\hline$-\mathrm{g} 2$ & $\mathbb{U}_{-1}$ & -6 & -5 & -4 & -3 & -2 & -1 & 0 & & & & $\lambda$ & 5 & 6 & $\mathbb{U}_{1}$ & $\mathrm{~g} 2$ \\
\hline$-\mathrm{g} 1$ & $\mathbb{U}_{-0}$ & -1 & -1 & -1 & -1 & -1 & -1 & 0 & & & $\Lambda$ & $x$ & 1 & 1 & $\mathbb{U}_{0}$ & $\mathrm{~g} 1$ \\
\hline g0 & \multicolumn{2}{|c|}{ Kernel } & \multicolumn{2}{|c|}{0} & 0 & 0 & -1 & 0 & 1 & 0 & 0 & 0 & 0 & \multicolumn{2}{|c|}{ Kernel } & g0 \\
\hline$-g(-1)$ & $\mathrm{un}_{-1}$ & 0 & 0 & 0 & 0 & 1 & -1 & 0 & 1 & -1 & 0 & 0 & 0 & 0 & $\mathrm{un}_{1}$ & $g(-1)$ \\
\hline$-g(-2)$ & $\mathbb{u}_{-2}$ & 0 & 0 & 0 & -1 & 2 & -1 & 0 & 1 & -2 & 1 & 0 & 0 & 0 & $\mathbb{u n}_{2}$ & $g(-2)$ \\
\hline$-g(-3)$ & $\mathbb{U}_{-3}$ & 0 & 0 & 1 & -3 & 3 & -1 & 0 & 1 & -3 & 3 & -1 & 0 & 0 & $\mathbb{u n}_{3}$ & $g(-3)$ \\
\hline$-g(-4)$ & un & 0 & -1 & 4 & -6 & 4 & -1 & 0 & 1 & -4 & 6 & -4 & 1 & 0 & $\mathrm{un}_{4}$ & $g(-4)$ \\
\hline$-g(-5)$ & $\mathbb{u}_{-5}$ & 1 & -5 & 10 & -10 & 5 & -1 & 0 & 1 & -5 & 10 & -10 & 5 & -1 & $\mathrm{an}_{5}$ & $g(-5)$ \\
\hline
\end{tabular}

flects on core problems that on the one hand it is a powerful and easy tool to quickly reflect if there is a solution but on the other hand it conceals a great deal of information. The fresh look at the table aims: (i) to reveal the "cogwheels" that make the method work; and (ii) probably the satisfaction of an easy solution has prevented curiosity to explore full potentials. This is illustrated by analysing: $\mathbb{U}_{1} \cdot \mathbb{U}_{2} \cdot \mathbb{U}_{3}$, in Table 3 .

Algebra does not call the sequence $\left\langle\begin{array}{llll}1 & 17 & 33 & 9\end{array}\right\rangle$ as the kernel; it does not explain the reasons for its order being 4; the sequences below the kernels, e.g. $\left\langle\begin{array}{lllll}1 & 16 & 16 & -24 & -9\end{array}\right\rangle$, are often looked at like an underworld; there is some knowledge of the diagonals but its composition is not common knowledge.

\subsection{Transforming Difference Tables into Conducemental Network}

$\mathrm{AC}$ is minded with explaining and unearthing the cogwheels of difference tables, whilst maintains the focus on single terms. Seven diagonals (alleles) in Table 3 are highlighted:

$$
\begin{aligned}
& \mathcal{A}_{0}=\left\{\begin{array}{lllllll}
1 & 23 & 133 & 331 & 410 & 250 & 60
\end{array}\right\} ; \quad \mathcal{A}_{1}=\left\{\begin{array}{llllll}
1 & 22 & 111 & 220 & 190 & 60
\end{array}\right\} ; \\
& \mathcal{A}_{2}=\left\{\begin{array}{lllll}
1 & 21 & 90 & 130 & 60
\end{array}\right\} ; \quad \mathcal{A}_{3}=\left\{\begin{array}{lllll}
1 & 20 & 70 & 60
\end{array}\right\} ; \quad \mathcal{A}_{4}=\left\{\begin{array}{llll}
1 & 19 & 51 & 9
\end{array}\right\} ; \\
& \mathcal{A}_{5}=\left\{\begin{array}{llll}
1 & 19 & 33 & 9
\end{array}\right\} ; \mathcal{A}_{6}=\left\{\begin{array}{llll}
1 & 18 & 33 & 9
\end{array}\right\} ; \text { Kernel }=\left\langle\begin{array}{llll}
1 & 17 & 33 & 9
\end{array}\right\rangle \text {. }
\end{aligned}
$$

The main features of the conducemental table include: i) degree, each value of which marks a hierarchical level; ii) ordinal, each value of which sets the identifier for the terms; its source term can start from any term and is an important one; any term prior to the source term is treated as zero; and iii) gradient, diagonal or alleles, as shown above. 
Table 3. Conducemental table or visual conducemental network for $\mathbb{U}_{1} \cdot \mathbb{U}_{2} \cdot \mathbb{U}_{3}$.

\begin{tabular}{|c|c|c|c|c|c|c|c|c|c|c|c|}
\hline 1 & & 1 & 2 & 3 & 4 & 5 & 6 & 7 & 8 & 9 & \multirow{4}{*}{ ס̊ } \\
\hline 2 & & 1 & 3 & 6 & 10 & 15 & 21 & 28 & 36 & 45 & \\
\hline 3 & & 1 & 4 & 10 & 20 & 35 & 56 & 84 & 120 & 165 & \\
\hline Degree & Allele & 0 & -1 & -2 & -3 & -4 & -5 & -6 & -7 & -8 & \\
\hline 6 & 0 & & 24 & 180 & 800 & 2625 & 7056 & 16,464 & 34,560 & \multicolumn{2}{|c|}{ Parent } \\
\hline 5 & 1 & & & & 620 & 1825 & 4431 & 9408 & 18,096 & 32,265 & \multirow{6}{*}{$\begin{array}{l} \\
0 \\
0 \\
\tilde{D} \\
0\end{array}$} \\
\hline 4 & 2 & 1 & & & 464 & 1205 & 2606 & 4977 & 8688 & 14,169 & \\
\hline 3 & 3 & 1 & 21 & NW & & & 1401 & 2371 & 3711 & 5481 & \\
\hline 2 & 4 & & 20 & 90 & 28 & & 660 & 970 & 1340 & 1770 & \\
\hline 1 & 5 & 1 & 19 & 70 & 130 & Nan & 250 & $3 \times 0$ & 370 & 430 & \\
\hline 0 & 6 & 1 & 18 & 51 & 60 & 60 & $6 Q$ & 60 & $\gamma Q$ & 60 & \\
\hline- & Kernel & 1 & 17 & 33 & 9 & 0 & 0 & 0 & 0 & \multicolumn{2}{|c|}{ Kernel } \\
\hline-1 & 7 & 1 & 16 & 16 & -24 & -9 & 0 & 0 & 0 & 0 & \multirow{5}{*}{ 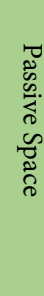 } \\
\hline-2 & 8 & 1 & 15 & 0 & -40 & 15 & 9 & 0 & 0 & 0 & \\
\hline-3 & 9 & 1 & 14 & -15 & -40 & 55 & -6 & -9 & 0 & 0 & \\
\hline-4 & 10 & 1 & 13 & -29 & -25 & 95 & -61 & -3 & 9 & 0 & \\
\hline-5 & 11 & 1 & 12 & -42 & 4 & 120 & -156 & 58 & 12 & -9 Base & \\
\hline
\end{tabular}

\subsection{Basic Operations Using Alleles}

Above alleles are interoperable (convertible to one another) through appropriate rules, e.g.:

$$
\begin{aligned}
\mathcal{A}_{0} & =\left\{\begin{array}{llllllll}
0 & 24 & 156 & 464 & 741 & 660 & 310 & 60
\end{array}\right\} \oplus\left\{\begin{array}{ccccccc}
1 & & & & & \\
-1 & 1 & 1 & & & & \\
1 & -1 & -1 & 1 & 1 & \\
-1 & 1 & 1 & -1 & -1 & 1 & 1 \\
1 & -1 & 1 & 1 & 1 & -1 \\
-1 & 1 & -1 & -1 & & \\
1 & -1 & & & &
\end{array}\right\} \\
& =\left\{\begin{array}{llllllll}
1 & 23 & 133 & 331 & 410 & 250 & 60
\end{array}\right\}
\end{aligned}
$$

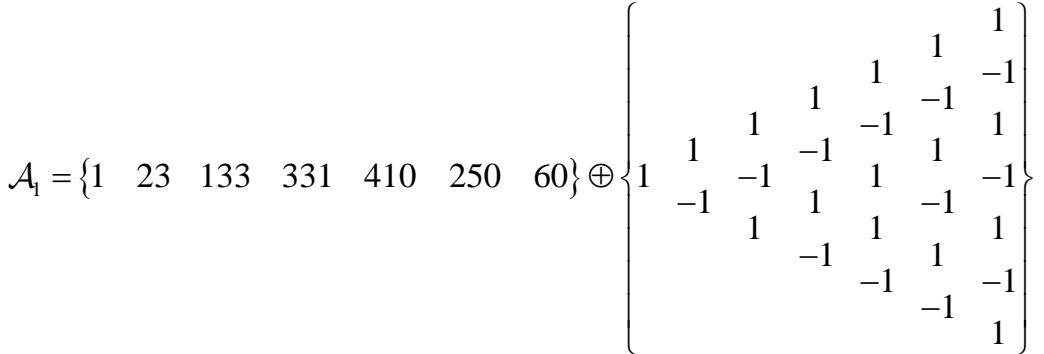

$$
\begin{aligned}
& =\left\{\begin{array}{llllll}
1 & 22 & 111 & 220 & 190 & 60
\end{array}\right\}
\end{aligned}
$$




$$
\begin{aligned}
& \mathcal{A}_{2}=\left\{\begin{array}{llllll}
1 & 22 & 111 & 220 & 190 & 60
\end{array}\right\} \oplus\left\{\begin{array}{cccccc} 
& & & & & 1 \\
& & 1 & 1 & & -1 \\
1 & & -1 & -1 & 1 \\
1 & -1 & 1 & 1 & -1 \\
-1 & 1 & -1 & 1 & 1 \\
& & & & -1 & -1
\end{array}\right\} \\
& =\left\{\begin{array}{lllll}
1 & 21 & 90 & 130 & 60
\end{array}\right\} \\
& \mathcal{A}_{3}=\left\{\begin{array}{lllll}
1 & 21 & 90 & 130 & 60
\end{array}\right\} \oplus\left\{\begin{array}{cccc} 
& & & \\
& 1 & 1 & -1 \\
1 & -1 & -1 & 1 \\
-1 & 1 & 1 & 1 \\
& & -1 & -1
\end{array}\right\} \\
& =\left\{\begin{array}{llll}
1 & 19 & 51 & 9
\end{array}\right\}
\end{aligned}
$$

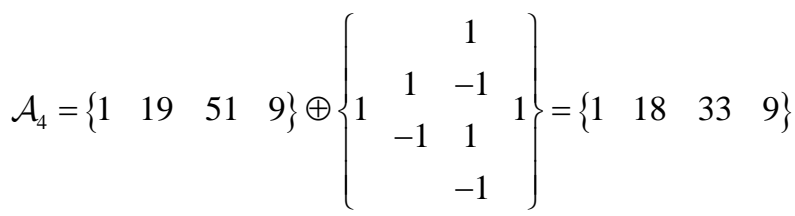

$$
\begin{aligned}
& \mathcal{A}_{5}=\left\{\begin{array}{llll}
1 & 18 & 33 & 9
\end{array}\right\} \oplus\left\{\begin{array}{ccc}
1 & 1 & 1 \\
-1 & 1
\end{array}\right\}=\left\{\begin{array}{llll}
1 & 17 & 33 & 9
\end{array}\right\} \\
& \mathcal{A}_{6}=\left\{\begin{array}{llll}
1 & 17 & 33 & 9
\end{array}\right\} \oplus\left\{\begin{array}{lllll}
1 & 1 & 1 & 1
\end{array}\right\}=\left\langle\begin{array}{lllll}
1 & 17 & 33 & 9
\end{array}\right\rangle
\end{aligned}
$$

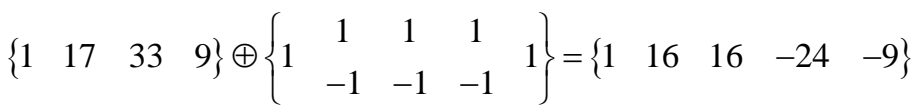

$$
\begin{aligned}
& \left\{\begin{array}{lllll}
1 & 16 & 16 & -24 & -9
\end{array}\right\} \oplus\left\{\begin{array}{cccccc}
1 & 1 & 1 & 1 & 1 & 1 \\
-1 & -1 & -1 & -1 & 1
\end{array}\right\} \\
& =\left\{\begin{array}{llllll}
1 & 15 & 0 & -40 & 15 & 9
\end{array}\right\}
\end{aligned}
$$

Remark 3.1: architectural variations in alleles are greyed, where these alleles initiate transient changes. AC makes these operations as routine but their algebraic operations are not.

\subsection{Morphology of Types 2 - 4 Conducemental Sequences}

The paper refers to all of (3.1) as operations on alleles, and to the enabler sequence in these operations as the sequence of counters, sequence of invariables, rules or replicators. These terms are regarded as synonymous but the term replicator is a curious one and easily graspable as these sequences are replications of the previous sub-terms plus introduction of a new sub-term.

The replicators in the operations seem to reveal the cogwheels but these are $\mathrm{AC}$ operations and do not exist in algebra. It should be mentioned that that these replicators undergo: (i) global (generic) variations highlighted in grey; and (ii) local (specific) variations. Global and local variations are a feature of each unbounded conducemental sequence, which are classified into types in Section 4.3. For instance, Type 2 displays the following characteristic feature with respect to 
their kernels:

$$
\begin{aligned}
& \mathbb{U}_{0} \cdot \mathbb{U}_{2} \cdot \mathbb{U}_{3}:\langle 1 \quad 6 \quad 3\rangle ; \omega=3 ; \delta=5 \\
& \mathbb{U}_{1} \cdot \mathbb{U}_{2} \cdot \mathbb{U}_{3}:\langle 1 \quad 17 \quad 33 \quad 3\rangle ; \omega=4 ; \delta=6 \\
& \mathbb{U}_{2} \cdot \mathbb{U}_{2} \cdot \mathbb{U}_{3}:\langle 1 \quad 28 \quad 100 \quad 72 \quad 9\rangle ; \omega=5 ; \delta=7 \\
& \mathbb{U}_{3} \cdot \mathbb{U}_{2} \cdot \mathbb{U}_{3}:\left\langle\begin{array}{llllll}
1 & 39 & 204 & 244 & 69 & 3
\end{array}\right\rangle ; \omega=6 ; ; \delta=8 \\
& \mathbb{U}_{4} \cdot \mathbb{U}_{2} \cdot \mathbb{U}_{3}:\langle 1 \quad 50 \quad 345 \quad 580 \quad 260 \quad 24\rangle ; \omega=6 ; \delta=9 \\
& \mathbb{U}_{5} \cdot \mathbb{U}_{2} \cdot \mathbb{U}_{3}:\langle 1 \quad 61 \quad 523 \quad 1135 \quad 700 \quad 100\rangle ; \omega=6 ; ; \delta=10
\end{aligned}
$$

It is observed that $\mathbb{U}_{2} \cdot \mathbb{U}_{3} \quad(\delta=5)$ is the core in (3.2) sequences; as its degree increases systematically (from 5 to 10 ), its order only increases up to $(\omega=6)$ but remains constant at 6 from $\mathbb{U}_{3} \cdot \mathbb{U}_{2} \cdot \mathbb{U}_{3}$ upwards. Contrast this with Type 4 sequences, as follows:

$$
\begin{aligned}
& \mathbb{B}_{1} \cdot \mathbb{B}_{2} \cdot \mathbb{B}_{1}:\left\langle\begin{array}{lllll}
1 & 32 & 62 & 0 & 1
\end{array}\right\rangle \omega=5 ; \delta=3 \\
& \mathbb{B}_{1} \cdot \mathbb{B}_{2} \cdot \mathbb{B}_{2}:\left\langle\begin{array}{lllll}
1 & 44 & 134 & 12 & 1
\end{array}\right\rangle \omega=6 ; ; \delta=3 \\
& \mathbb{B}_{1} \cdot \mathbb{B}_{2} \cdot \mathbb{B}_{3}:\left\langle\begin{array}{llllll}
1 & 56 & 246 & 116 & -47 & 12
\end{array}\right\rangle \omega=6 ; \delta=3 \\
& \mathbb{B}_{1} \cdot \mathbb{B}_{2} \cdot \mathbb{B}_{4}:\left\langle\begin{array}{lllllll}
1 & 68 & 398 & 396 & -47 & -88 & 40
\end{array}\right\rangle=7 ; \delta=3
\end{aligned}
$$

It is further noted that the order of $\mathbb{B}_{1}, \mathbb{B}_{2}, \mathbb{B}_{1}$ and $\mathbb{B}_{1} \cdot \mathbb{B}_{2}, \mathbb{B}_{2}$ remains at the constant value of 5 but increases by " 1 " from $\mathbb{B}_{1}, \mathbb{B}_{2} \cdot \mathbb{B}_{3}$ upwards, while their degree remains at 3. In this way, the morphology of Type 4 sequences is in a sharp contrast with those of Type 2 sequences. These morphological differences are not accidental or arbitrary but this paper only draws attention to the differences in these features, which are determinable from their dimensional specifications, but they will be analysed in separate papers. These morphological architectures are not trivial but they allow a complete regeneration of each and all conducemental sequences. These are explained heuristically in the next section.

The morphology of each conducemental sequence is determined by its order. Consider the morphology of some of the simple conducemental sequences of Types 2 to 4 in Tables 4(a)-(c). The paper expressly states that all the variations in the order and degree (architectural specification) are determinate in terms of dimensional specification and the values shown in Tables 4(a)-(c) exemplify the problem.

\section{Classifying Conducemental Sequences-Functionology}

The paper is indirectly concerned with generating functionology [4], which transforms any algebraic functions to polynomial functions. However, the paper employs none of these techniques and offers a fresh approach to the subject, which is analytical.

Convolution products have an interesting structure but conventional algebra conceals them by expressing the products in terms of algebraic functions in- 
cluding polynomial functions but conducemental algebra unearths their structures. The paper unearths these structures in terms of their degree and order by the following classification: Type 1: the degree of the sequence is between $-\infty$ and $\infty$ but its order is constant; Type 2: $\delta \geq \omega$ plus other requirements; Type 3: $\delta>\omega$ plus other requirement and Type 3: $\delta<\omega$ plus other requirements. These are outlined in this section.

\subsection{Overview of Morphology of Sequences}

A glimpse of morphology is already presented in Section 3.4 indicates that a serious variability in the general morphology of conducemental sequences. Consider Table 4(a) \& Table 4(c).

Table 4. (a) Reducementals of simple unbounded conducemental sequences: $\delta \geq \omega$; (b) reducementals of regenerated unbounded conducemental sequences: $\delta \geq \omega$; (c) reducementals for a set of unbounded conducemental sequences.

(a)

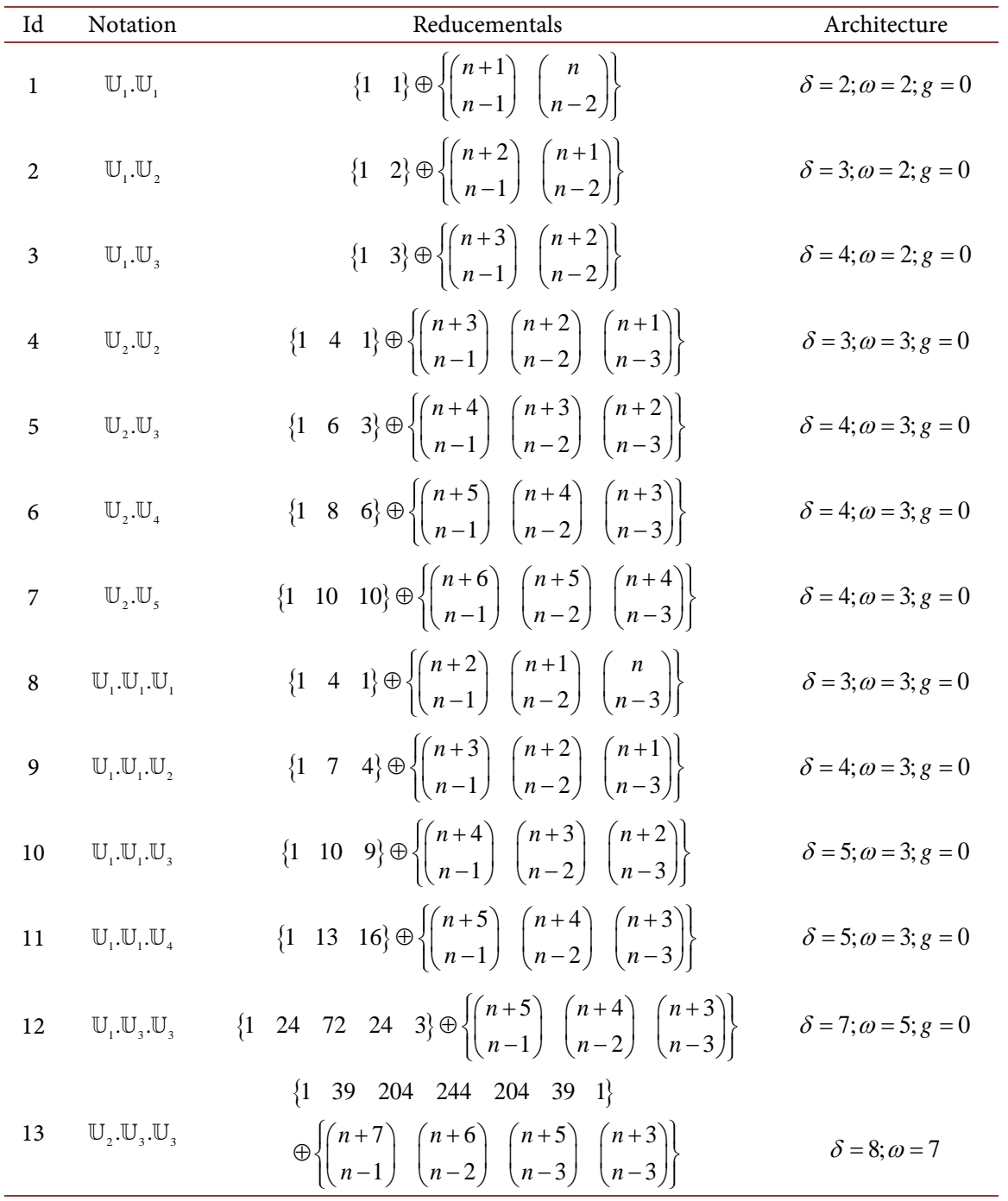

Remark 4.1: The sequence $\left(\mathbb{U}_{1} \cdot \mathbb{U}_{1} \cdot \mathbb{U}_{1}\right)$, ID 8, is Example 6.1 for a more detailed study. 
(b)

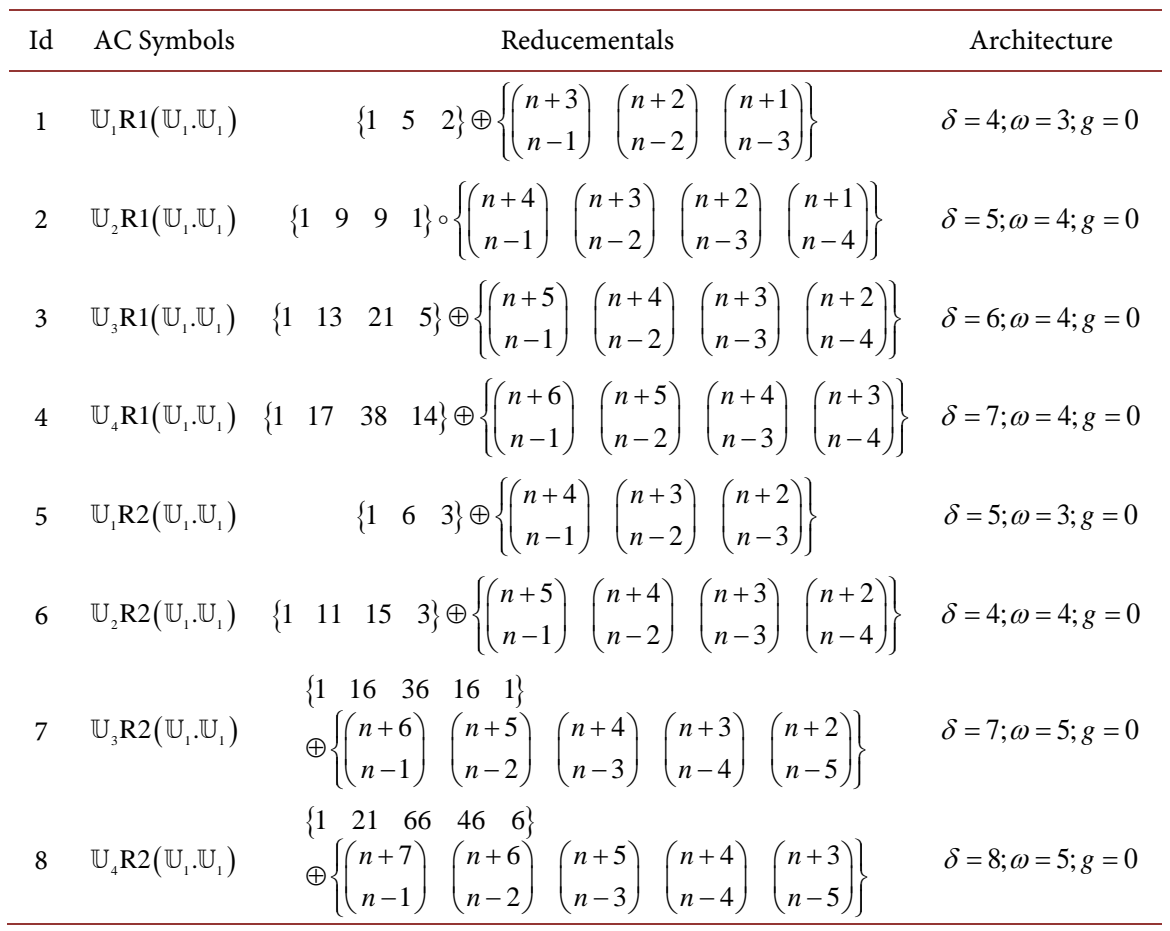

Remark 4.2: $\mathrm{R} 1$ denotes regeneration once, $\mathrm{R} 2$ denotes regeneration twice and so one.

(c)

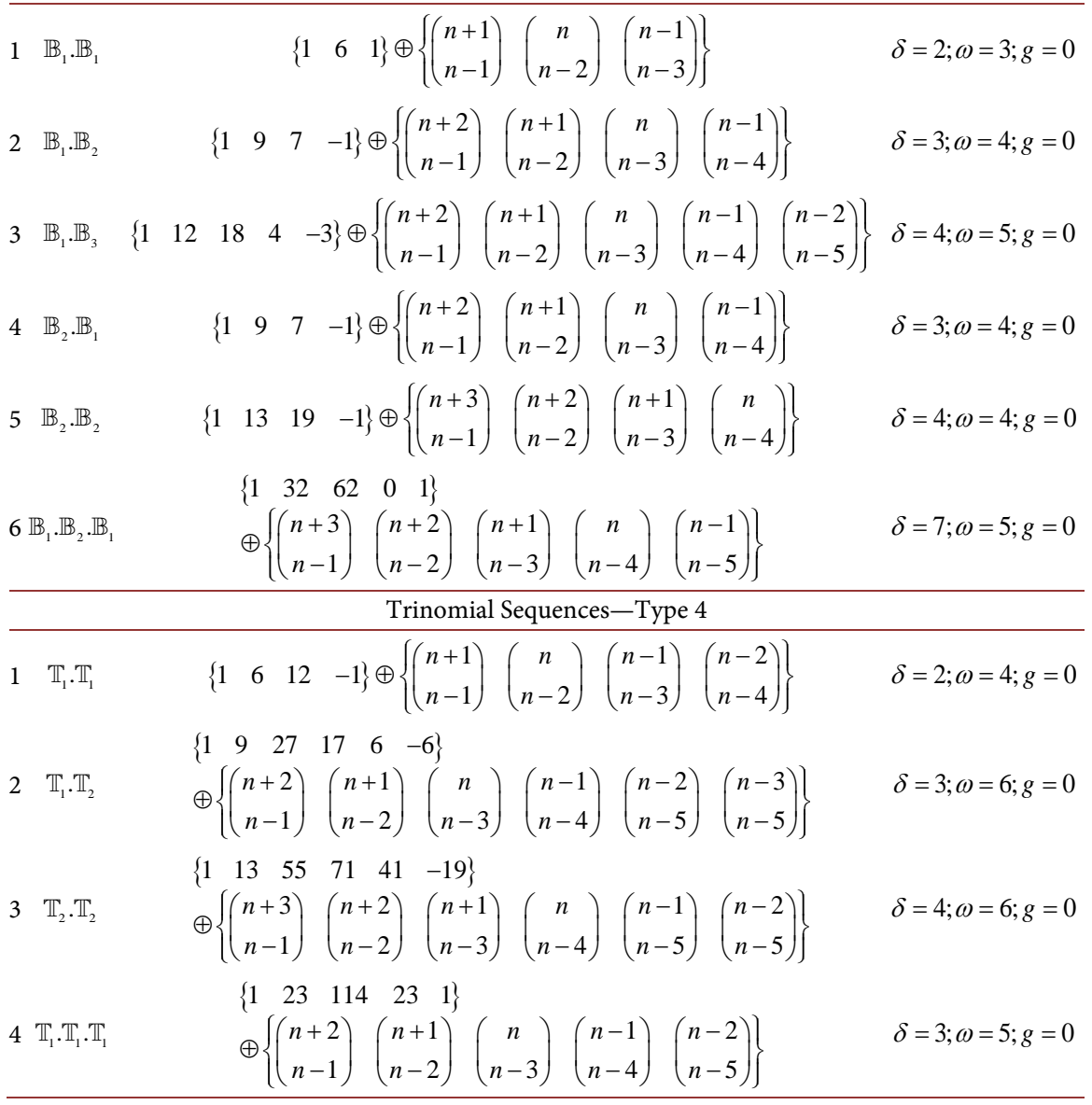


The variability in the order of the examples in Table 4(a) \& Table 4(b) need to be explained. The author has analysed literally thousands of such sequences to understand the underlying patterns. Although these patterns will be explained in a future paper, it suffices to mention that they are determinate but the way out is to classify these sequence, as given in Section 4.3.

\subsection{Architecture of Conducemental Sequences}

Tables 4(a)-(c) reflect on the morphology of unbounded conducemental sequences; which are implicitly captured in Table 3 but captured more explicitly in Table 5.

Table 5 provides a visual aid to draw attention to a network in which the players are: parent sequences, base sequences, degrees/hierarchies in the vertical sense, ordinal in the horizontal sense, alleles along the diagonal; active and passive spaces and the kernel space; generic variations in the allele zones treated by a route map but not detailed in the paper.

\subsection{Classification of Unbounded Conducemental Sequences}

The whole conducemental sequences are classified into five types of conducemental sequences. Types 1 - 4, defined below, are to be referred to as unbounded conducemental sequences but Type 5 , as bounded conducemental sequences, are only outlined.

\subsubsection{Type 1 Sequences}

This type of sequences are the regeneration products of generations of sequences

Table 5. Bird's eye of view of architecture of conducemental networks.

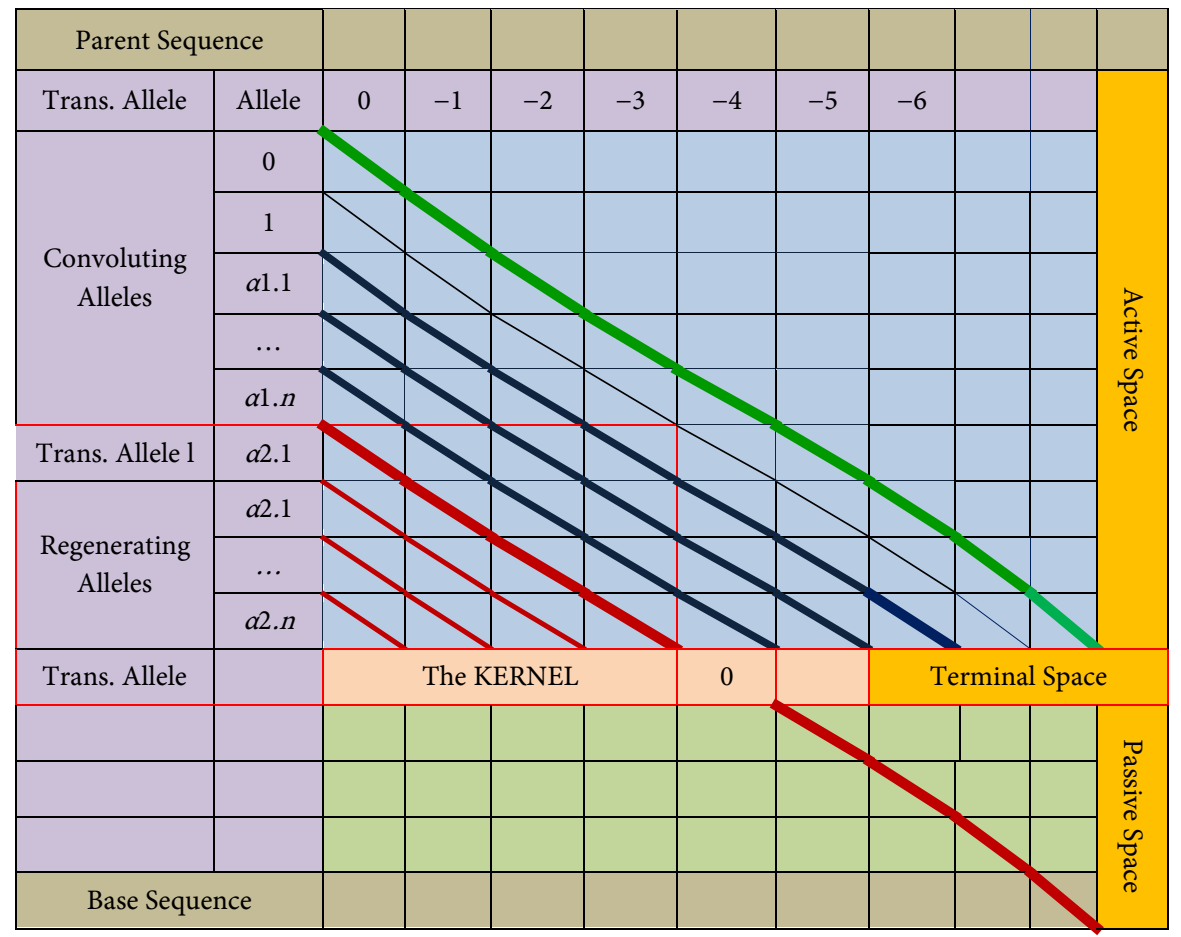


based on natural numbers. These are referred to as the uninomial sequences, defined as follows:

$$
\left\{\text { Cond. } \mid\{\overbrace{\mathbb{S}\}}^{d}\}=\left\{\text { Cond. } \mid\{\overbrace{\mathbb{N}\}}^{d_{1}} \oplus \overbrace{\{\mathbb{N}\}}^{d_{2}} \oplus \overbrace{\{\mathbb{N}\}}^{d_{3}} \oplus \cdots \oplus \overbrace{\{\mathbb{N}\}}^{d_{i}}\}=\{1\} \odot\left\{\left(\begin{array}{c}
n+d \\
n-1
\end{array}\right)\right\}\right.\right.
$$

where, $d=d_{1}+d_{2}+d_{3}+\cdots+d_{i}$; The expression in (4.1) is read as the conducement of Sequence $\mathbb{S}$ of degree $d$ is given by the convolution products of natural number of degrees of $d_{1}, d_{2}, d_{3}, \cdots$ and $d_{i}$ and its conducemental is given by the product of two sequences, as shown. The dimensional specification of Type 1 sequences is:

$$
\{\mathcal{D S} \mid \mathbb{S}\}=\left\lceil\langle\mathcal{A S} \mid d, 1, d+1\rangle\left\langle\mathcal{L S} \mid 1, \varnothing_{a} \quad d_{f}, d_{t}\right\rangle \text { Defect }\langle\mathcal{K} \mid 1\rangle\right\rceil
$$

Remark 4.3: Dimension specification of uninomial unbounded sequence is:

$$
\left\lceil\mathcal{D S}\left|\quad \mathbb{U}_{1}\right| \begin{array}{llll}
1 & 1 & \cdots
\end{array}\right]=\lceil\langle\mathcal{A S} \mid 0,1,1\rangle\langle\mathcal{L S} \mid 1\rangle \quad \text { Defect }\langle\mathcal{K} \mid 1\rangle\rceil
$$

Its inverse is:

$$
\left\{\mathcal{D S} \mid \mathfrak{\varpi}_{-1}\right\}=\lceil\langle\mathcal{A S} \mid-1,1,1\rangle\langle\mathcal{L S} \mid 1\rangle \quad \text { Defect }\langle\mathcal{K} \mid 1\rangle\rceil
$$

Remark 4.4: For each multiplication by $\left\{\begin{array}{lll}1 & 1 & \cdots\end{array}\right\} \oplus$, the degree is increased by "1".

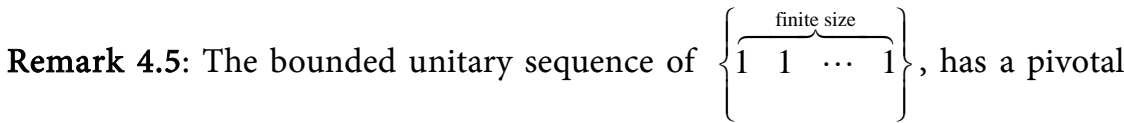
role in Type 4 sequences; the unbounded unitary sequence, $\left\{\begin{array}{lllll}1 & 1 & 1 & 1 & \ldots\end{array}\right\}$ cannot be used with the convolution products rule, $\hat{\oplus}$; and it has no effect when used in direct product with $\circ$.

\subsubsection{Type 2 Sequences}

This type of sequences is the direct products of Type 1 sequences, expressed as follows:

$$
\begin{aligned}
& \left\{\operatorname{Red} \mid\{\overbrace{\substack{\mathbb{S} \\
\omega}}^{d}=\{\overbrace{\left\{\underset{\omega_{1}}{\mathbb{S}}\right\}}^{d_{1}} \circ \overbrace{\left\{\begin{array}{c}
\mathbb{S} \\
\omega_{\omega_{2}}
\end{array}\right\}}^{d_{2}} \circ \cdots \circ \overbrace{\left\{\begin{array}{c}
\mathbb{S} \\
\omega_{i}
\end{array}\right\}}^{d_{i}}\}\}\right. \\
& =\left\{\begin{array}{llll}
T_{1} & T_{2} & \cdots & T_{\omega}
\end{array}\right\} \odot\left\{\left(\begin{array}{c}
n+d-1 \\
n-1
\end{array}\right)\left(\begin{array}{c}
n+d-2 \\
n-2
\end{array}\right) \cdots\left(\begin{array}{c}
n+d-\omega \\
n-\omega
\end{array}\right)\right\}
\end{aligned}
$$

where $d_{1}, d_{2}, d_{3}, \cdots, d_{i}$, are the degrees of the individual sequences and $T$ is the values of the terms of the kernels from 1 to $\omega$. Notably, (4.3a) is read as: the reducemental of Sequence $\mathbb{S}$ of degree $d$. The degree at its kernel is given by the direct products of lower sequences of degrees $d_{1}, d_{2}, d_{3}, \cdots$ and $d_{i}$ with corresponding orders of $\omega_{1}, \omega_{2}, \omega_{3}, \cdots$ and $\omega_{i}$ ), in which the following hold:

$$
d=d_{1}+d_{2}+d_{3}+\cdots+d_{i}
$$

The order of the Type 2 conducemental sequences is determined as follows: 


$$
\omega=d_{1}+d_{2}+d_{3}+\cdots+d_{i}-\max \left(d_{1}, d_{2}, d_{3}, \cdots, d_{i}\right)+1
$$

The dimensional specification of Type 2 sequences is:

$$
\{\mathcal{D S} \mid \mathbb{S}\}=\left\lceil\langle\mathcal{A S} \mid \delta, \omega, 0\rangle\langle\mathcal{L S} \mid 1\rangle \quad \text { Defect } \quad\left\langle\mathcal{K} \mid T_{1} \quad T_{2} \quad \cdots \quad T_{\omega}\right\rangle\right\rceil
$$

\subsubsection{Type 3 Sequences}

Conducemental sequences of Types 1 and 2 may be combined, so that the overall product includes any number of both direct and regeneration product rules.

Example 4.1: Consider the example:

$$
\{\{\overbrace{\{\mathbb{S}\}}^{d_{1}} \circ \overbrace{\{\mathbb{S}\}}^{d_{2}}\} \oplus \overbrace{\{\underbrace{\mathbb{U}\}}_{g=1}\}}^{d} \circ \overbrace{\{\mathbb{S}\}}^{d_{3}} \circ \cdots \circ \overbrace{\{\mathbb{S}\}}^{d_{i}}
$$

(4.4a) is a conglomerate; its degree is the sum of the degree of individual constituents; its order is determinate but complex and not presented here; and its dimensions are specified:

$$
\{\mathcal{D S} \mid \mathbb{S}\}=\left\lceil\langle\mathcal{A S} \mid \delta, \omega, g\rangle\left\langle\langle\mathcal{L S} \mid 1\rangle \quad \text { Defect } \quad\left\langle\mathcal{K} \mid T_{1} \quad T_{2} \quad \cdots \quad T_{\omega}\right\rangle\right\rceil\right.
$$

where $\delta, \omega$ have definitive values but follow complex rules not given in the paper.

\subsubsection{Type 4 Sequences}

These types of conducemental sequences comprise any number of conglomerate constituents, which involve at least one convolution product. One example is as follows:

$$
\left.\left\{\{\overbrace{\{\mathbb{S}\}}^{d_{1}}\} \oplus\{u\}\right\} \circ\{\overbrace{\{\mathbb{S}\}}^{d_{2}}\} \oplus\{u\}\right\} \circ \cdots \circ\left\{\{\overbrace{\{\mathbb{S}\}}^{d_{i}}\} \oplus\{u\}\right\}
$$

where $\{u\}$ is a bounded sequence of counter, e.g. $\left\{\begin{array}{ll}1 & 1\end{array}\right\}$ or $\left\{\begin{array}{lll}1 & 1 & 1\end{array}\right\}$. In pure Type 4 multiplications, the degrees are not affected but the kernel orders are calculated as:

$$
\begin{aligned}
d_{\max } & =\left\{\max \mid d_{1}, d_{2}, d_{3}, \cdots, d_{i}\right\} \\
\sigma & =d_{\max }+1_{\text {for each multiplication }}
\end{aligned}
$$

The conducemental dimensional specification of Type 4 sequences is:

$$
\{\mathcal{D S} \mid \mathbb{S}\}=\left\lceil\langle\mathcal{A S} \mid \delta, \omega, 0\rangle\langle\mathcal{L S} \mid 1\rangle \quad \text { Defect } \quad\left\langle\mathcal{K} \mid T_{1} \quad T_{2} \quad \cdots \quad T_{\sigma}\right\rangle\right\rceil
$$

\subsection{Bounded Conducemental Sequences-Type 5}

Both kernels and sequence of counters are bounded and their values may even be selected at random but operated by the conducemental algebra. These are outside the remit of the paper but they are also classified and some of these classes correspond to exponential series of the type $\left(n^{m}\right)$, e.g. the sequences in Pascal Triangle, which corresponds to $2^{m}$. Notably, Examples $1.1-1.4$ in Introduction are related to Type 5 sequences. 


\subsection{Further Definitions on Unbounded Conducemental Sequences}

Further definitions are necessary to capture the information conveyed in Table 3 and Table 4 but the intention is not to present rigorous definitions at this initiation stage. As shown in these tables, the domain of a parent sequence is divided into three spaces: (i) active space, (ii) kernel space and (iii) passive space. The AC way of thinking should be organised in two ways: (i) the problems are stated within a range of hierarchies (degrees): From the top of the range (parent sequence) to the bottom of the range (the base sequence) in the reducement problems or vice versa (from the base to the parent sequences) in the conducement problems; (ii) the focus of operations is on individual terms; and (iii) these spaces make up a new network to be referred to as the conducemental network. The passive space is the inverse of the active space and conjugate of each other but separated by the kernel space.

The overview of the conducemental network is as follows. (i) Active spaces are under direct attention and hold the hierarchies, each of which has positive values of a degree. (ii) Each hierarchy in this domain is a regeneration of its immediately lower hierarchy and therefore one degree more than it. (ii) The information content extractable from a hierarchy is only limited to the value of its degree. (iii) More information is contained in the gradients (alleles or diagonals) of the hierarchies ranging from the parent sequence to its kernel zone, as the diagonals retain sufficient information in the form of bounded conducemental sequences for the regeneration of the parent sequence and thereby of each and all of the hierarchies. (iv) Passive spaces are conjugates of active spaces, i.e. given one the other is available whether wanted or not. (vi) Each row in the passive space is a bounded sequence like the diagonals and has the dual function of acting as a diagonal and a hierarchy. The terms gradient, diagonal and allele are synonymous but diagonal or gradient in the passive space is meaningless as in the passive space, where both sequences and diagonals are coincident.

Degree, order and ordinal are three fundamental parameters to specify any parent sequence. (i) Degree is a fundamental property and defines a hierarchy; (ii) Each and every hierarchy of a parent sequence has the same order and as such order is a fundamental property similar to degree. (iii) The ordinal of the first term of a parent sequence is an independent variable and must be specified, as this affects the alleles.

\section{Morphology of Type 1 Conducemental Sequences}

The following three terms needs to be emphasised: (i) morphology refers to visible features of the reducementals in terms of order, alleles, degree and source term; (ii) architecture refers to the role of morphological features shared across the types of conducemental sequences; and (iii) structure refers to the layout of building blocks within each term.

Morphology of Type 1 unbounded sequences, given in Table 1, is the simplest of these unbounded conducemental sequences and presented in this section to 
lay the ground for morphology and structure but architecture will have some coverage in Section 6.

\subsection{Reducemental of Type 1 Sequences}

Proposition 5.1: Uninomial unbounded sequences, $\mathbb{U}_{d}$ of degree $d$ is factorised into the direct products of: (i) the sequence of building blocks and the sequence of counters.

$$
\begin{aligned}
& \left\{\left(\begin{array}{l}
d \\
0
\end{array}\right)\left(\begin{array}{l}
d \\
1
\end{array}\right)\left(\begin{array}{l}
d \\
2
\end{array}\right) \cdots\left(\begin{array}{l}
d \\
d
\end{array}\right)\right\} \odot\left\{\left(\begin{array}{l}
n \\
n
\end{array}\right)\left(\begin{array}{c}
n \\
n-1
\end{array}\right)\left(\begin{array}{c}
n \\
n-2
\end{array}\right) \cdots\left(\begin{array}{c}
n \\
n-d
\end{array}\right)\right\} \\
& =\left(\begin{array}{c}
n+d \\
n
\end{array}\right)
\end{aligned}
$$

The proposition is well known in algebra and no need to prove it. The only difference between (5.1) and those in algebra is the notation used but this is not trivial as it opens up the methodology for interpreting the problem. Thus, Type 1 unbounded sequences show that each term is an arrangement of the building block expressed in terms of degree $\left(\left\{\left(\begin{array}{l}d \\ 0\end{array}\right)\left(\begin{array}{l}d \\ 1\end{array}\right)\left(\begin{array}{l}d \\ 2\end{array}\right) \cdots\left(\begin{array}{l}d \\ d\end{array}\right)\right\}\right)$ based on the rule for object selection without replacement, as an expression of a self-replication process described later.

\subsection{Rule Base for Layout of the Building Blocks}

Type 1 Unbounded Conducemental Sequences and their layout in terms of the building blocks is outlined here to unearth the morphology within conducemental sequences, see also [1]. A set of rule-base is needed, as given in Table 6 .

\subsection{AC Treatment of Type 1 Uninomial Sequences}

\subsubsection{Processing Generation 0 -The Priming Operation}

The sequence is processed as follows:

The kernel $\langle 1\rangle$ is transformed into a sequence by "priming", as follows:

$$
\langle 1\rangle \circ \overbrace{\{1\}}^{\text {given rule }}=\{1\}
$$

The primed (5.2) is the uninomial bounded sequence of Generation 0 and yet has no degree. It serves as the building block for sequences of higher generations.

\subsubsection{Processing Generation 1}

i) Generation 1 sequence is generated as follows:

$$
\left.\mathbb{U}_{0}=\widehat{\{1}\right\} \oplus \overbrace{\left\{\begin{array}{lllll}
1 & 1 & 1 & 1 & \cdots
\end{array}\right\}}^{\text {unitary sequence }}=\underbrace{\overbrace{\begin{array}{lllll}
1 & 1 & 1 & 1 & \cdots
\end{array}}^{\text {product }}}_{\text {Generation } 1}
$$

ii)Dimensional Specification:

$$
\left\{\mathcal{D S} \mid \mathbb{U}_{0}\right\}=\lceil\langle\text { as } \mid 0,1,1\rangle \quad\langle\text { ls } \mid 1\rangle \quad \text { Defect } \quad\langle\mathcal{K} \mid 1\rangle\rceil
$$


Table 6. Rules for the structure of hierarchies based on uninomial sequences.

Rule Description

Framework: Type 1 Uninomial Unbounded Conducement Sequences

Remark 5.1: ST: Sub-Term; SST: Sub-sub-term; SSST: Sub-sub-sub-term

5.1 Each sequence at a hierarchy is unbounded and the identity of its terms is identified by the ordinal of the terms (e.g. $3^{\text {rd }}$ ).

5.2 Generation $G=1(d=0)$ : each term is composed of one term in its internal structure

5.3 Generation $G=2(d=1)$ : each term has as many ST as its ordinal number, e.g. Term 3 has 3 sub-terms.

5.4 Generation $\boldsymbol{G}=\mathbf{3}(\boldsymbol{d}=2)$ : each ST has as many SST as its ordinal number.

5.5 Generation $\boldsymbol{G}=\mathbf{4}(\boldsymbol{d}=\mathbf{3})$ : each SST has as many SSST as its ordinal.

5.6 Rules (5.1)-(5.6) are recursive with the effect of nesting within each hierarchy the structures of the numbers at its lower hierarchy-the regeneration product rule.

\section{Generalisation: Types 2, 3 and 4 Unbounded Conducemental Sequences}

Type 1 uninomial sequences all have the kernel of: $\langle 1\rangle$ but the kernel size of Types 2, 3 and 4

5.7 parent sequences have the kernel: $\left.\begin{array}{llllll}T_{1} & T_{2} & T_{3} & \cdots & T_{\omega}\end{array}\right\rangle$, with their sizes, $\omega$, may be less than, equal to and greater than the degree of parent sequences.

The first term of the parent sequence will replace $\langle 1\rangle$ with $T_{1}$, the second term will replace that with: $T_{1}+T_{2}$; the third term with: $T_{1}+T_{2}+T_{3}, \cdots$ and the $\omega^{\text {th }}$ term with: 5.8 $T_{1}+T_{2}+T_{3}+\cdots+T_{\omega}$. Thereafter, the kernel will be replicated as a whole, i.e. for $(\omega+1)^{\text {th }}$ term, there is one additional term of $T_{1}+T_{2}+T_{3}+\cdots+T_{\omega}$ and for $(\omega+2)^{\text {th }}$ term, there are two additional terms of $T_{1}+T_{2}+T_{3}+\cdots+T_{\omega}$ and so on.

Remark 5.2: Attention is drawn to the deep level of replication in this rule table.

iii) The product is Type 1 unbounded conducemental sequence, expanded as per Rules 5.3:

$$
\underbrace{\underbrace{d=0}_{\left.\mathbb{U}_{0}\right\}}}_{g=1}=\left\{\begin{array}{lllll}
1 & 1 & 1 & 1 & \cdots
\end{array}\right\}=\left\{\begin{array}{ccccc}
T 1 & T_{2} & T 3 & T 4 & \\
\hat{1} & \overrightarrow{1} & \overrightarrow{1} & \hat{1} & \cdots \\
& & & &
\end{array}\right\}
$$

\subsubsection{Processing Generation 2-Natural Numbers}

The product of (5.3c) serves as building blocks for the sequences of higher degrees and in this case conducing from Generation 1 uninomial sequence using the unitary sequence produces natural numbers:

(i)

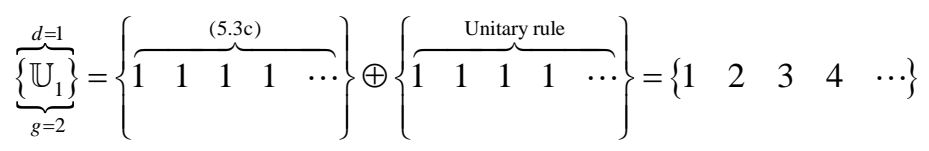

(ii) Dimensional Specification:

$$
\left\{\mathcal{D S} \mid \mathbb{U}_{0}\right\}=\lceil\langle a s \mid 1,1,2\rangle \quad\langle l s \mid 1\rangle \quad \text { Defect }\langle\mathcal{K} \mid 1\rangle\rceil
$$

(iii) The product is the sequence of natural numbers, expanded per Rules 5.4 of Table 6: 


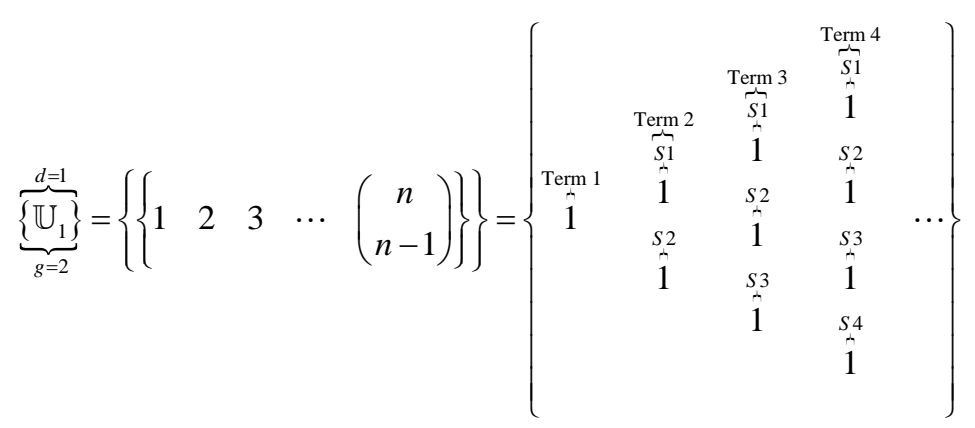

(iv) The reducemental of $\mathbb{U}_{1}$ as per Proposition 5.1 is given by:

$$
\left\{\left(\begin{array}{l}
1 \\
0
\end{array}\right)\left(\begin{array}{l}
1 \\
1
\end{array}\right)\right\}\left\{\left(\begin{array}{l}
n \\
n
\end{array}\right)\left(\begin{array}{c}
n \\
n-1
\end{array}\right)\right\}
$$

Remark 5.3: (5.4d) has the allele of $\left\{\begin{array}{ll}1 & 1\end{array}\right\}$ and this is shown as a diagonal in Table 2 but associated with a different replicator based on object selection without replacement.

\subsubsection{Processing Generation 3, Degree 2}

(i) Uninomial sequence is conduced to Generation 3 from Generation 2, as follows:

$$
\underbrace{\left\{\mathbb{U}_{2}\right\}}_{g=3}\}=\left\{\begin{array}{lllll}
1 & 3 & 6 & 10 & \cdots
\end{array}\right\} \oplus\left\{\begin{array}{lllll}
1 & 1 & 1 & 1 & \cdots
\end{array}\right\}=\left\{\begin{array}{llllll}
1 & 4 & 10 & 20 & \cdots
\end{array}\right\}
$$

(ii) Dimensional Specification:

$$
\left\{\mathcal{D S} \mid \mathbb{U}_{2}\right\}=\left\lceil\langle a s \mid 2,1,3\rangle \quad\left\langle l_{s} \mid 1\right\rangle \quad \text { Defect }\langle\mathcal{K} \mid 1\rangle\right\rceil
$$

(iii) The product is a Type 1 sequence and is expanded per Rules 5.4, as follows:

(iv)

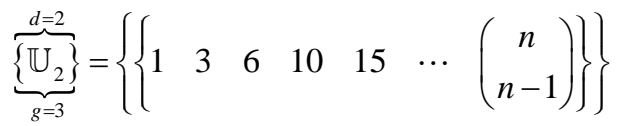

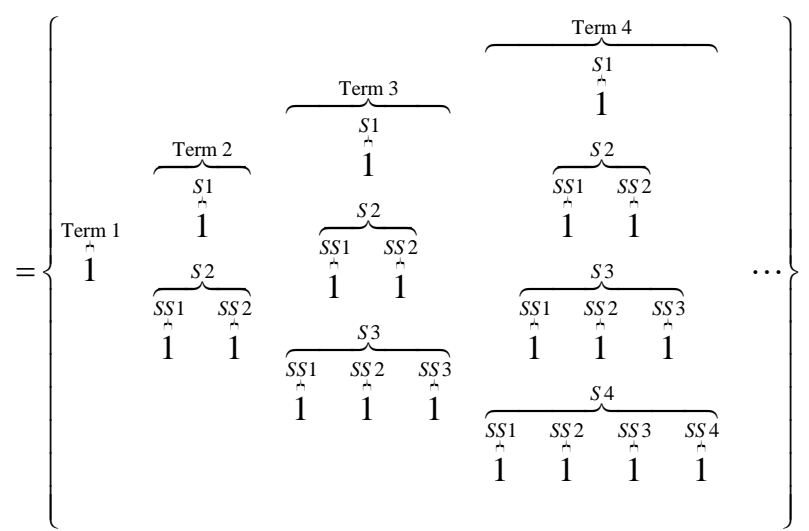

Elegant replication processes in $(5.5 \mathrm{c})$ are visually apparent and it can be viewed as follows: it is clear that (5.4c) is nested in (5.5c); and each term in (5.5c) is a replication of its previous terms plus one new term, in which the kernel will 
supply its values.

(v) The reducemental of $\mathbb{U}_{2}$ is given by:

$$
\left\{\left(\begin{array}{l}
2 \\
0
\end{array}\right)\left(\begin{array}{l}
2 \\
1
\end{array}\right)\left(\begin{array}{l}
2 \\
2
\end{array}\right)\right\} \odot\left\{\left(\begin{array}{l}
n \\
n
\end{array}\right)\left(\begin{array}{c}
n \\
n-1
\end{array}\right)\left(\begin{array}{c}
n \\
n-2
\end{array}\right)\right\}
$$

Remark 5.4: (5.5d) has allele of $\left\{\begin{array}{lll}1 & 2 & 1\end{array}\right\}$ and this is shown as a diagonal in Table 2 but associated with a different replicator based on object selection without replacement.

\section{Types 2 Unbounded Sequence-Tentative Investigation}

Each sequence in the AC space has countless expressions, all of which may be transformed into a single equivalent expression in the algebraic space. For instance, arithmetic progression is expressed algebraically as $2 n \pm 1$ but there are countless AC expressions for this. Examples include:

$$
\begin{aligned}
& \{1 \quad 1\} \odot\left\{\left(\begin{array}{c}
n \\
n-1
\end{array}\right)\left(\begin{array}{l}
n-1 \\
n-2
\end{array}\right)\right\}, \quad\{1 \quad 2\} \odot\left\{\left(\begin{array}{l}
n-1 \\
n-1
\end{array}\right)\left(\begin{array}{l}
n-1 \\
n-2
\end{array}\right)\right\} ; \\
& \{3 \quad 2\} \odot\left\{\left(\begin{array}{l}
n-2 \\
n-2
\end{array}\right)\left(\begin{array}{l}
n-2 \\
n-3
\end{array}\right)\right\}, \quad\left\{\begin{array}{ll}
3 & -1
\end{array}\right\}\left\{\left(\begin{array}{l}
n-1 \\
n-2
\end{array}\right)\left(\begin{array}{l}
n-2 \\
n-3
\end{array}\right)\right\} \text { and countless oth- }
\end{aligned}
$$

ers.

Similarly, $n^{3}$ is a single algebraic expression for a single sequence but this may be expressed by AC expressions capturing each idiosyncratic variation. The expressions in both spaces are apparently the same in numerical terms by-andlarge but there are differences between them including: (i) the sequences in the $\mathrm{AC}$ spaces have all real values for each value of the sequence of counters but their algebraic expression for some of these sequences suffer from singularity points (although no example is given to this problem due to the remit of the paper; and (ii) AC exposes various types of architectures and structures, which are otherwise concealed in the algebraic space.

The paper breaks down conducemental algebra to the following distinct bottomup problem areas: (i) reducement from one hierarchy to a lower hierarchy (ii) conducement problems from a lower to a higher hierarchy, (iii) gradient operations - hierarchies/alleles operations; (iv) allele-to-allele operations both reducement and conducement; and (vi) interoperability operations; and (vii) further operations on structure of hierarchies.

\subsection{Methodology for Conducement/Reducement}

As a pretext to $\mathrm{AC}$, it is stated that every problem in $\mathrm{AC}$ follows generic operators. The above problems are presented fully in Table 7 for Type 2 sequences through the following example:

Example 6.1: Use a Type 2 sequence as follows:

$$
\mathbb{U} 1 . \mathbb{U} 1 . \mathbb{U} 1=\{\mathbb{S}\}=\left\{\begin{array}{llllll}
1 & 8 & 27 & 64 & 125 & \cdots
\end{array}\right\}
$$

Dimensional Specification: 
Table 7. Tabular representation of Example 6.1 for reference purposes.

\begin{tabular}{|c|c|c|c|c|c|c|c|c|c|c|c|c|}
\hline \multicolumn{2}{|c|}{ Ordinals $\rightarrow$} & 1 & 2 & 3 & 4 & 5 & 6 & 7 & 8 & 9 & \multicolumn{2}{|c|}{10} \\
\hline Deg & Alleles & 0 & -1 & -2 & -3 & -4 & -5 & -6 & -7 & -8 & & -9 \\
\hline 3 & 0 & & & 27 & 64 & 125 & 216 & 343 & 512 & \multicolumn{3}{|c|}{ Parent S. } \\
\hline 2 & 1 & & & & J & 61 & 91 & 127 & 169 & 217 & \multirow{3}{*}{ 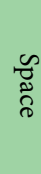 } & \multirow{3}{*}{ 常 } \\
\hline 1 & 2 & & & & 18 & 24 & 30 & 36 & 42 & 48 & & \\
\hline 0 & 3 & 1 & & & & & 6 & 6 & 6 & 6 & & \\
\hline - & 4 & 1 & 4 & & 0 & 0 & & & & \multicolumn{3}{|c|}{ Kernel Space } \\
\hline-1 & 5 & 1 & 3 & -3 & -1 & 0 & 0 & 0 & 0 & 0 & \multirow{3}{*}{$\begin{array}{l}\widetilde{D} \\
\text { గై }\end{array}$} & \multirow{3}{*}{ 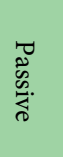 } \\
\hline-2 & 6 & 1 & 2 & -6 & 2 & 1 & 0 & 0 & 0 & 0 & & \\
\hline-3 & 7 & 1 & 1 & -8 & 8 & -1 & -1 & 0 & \multicolumn{2}{|c|}{ Base S. } & & \\
\hline
\end{tabular}

\subsection{Problem of Reducement/Conducement between Hierarchies}

\subsubsection{Problem of Reducement from one Hierarchy to Another}

Proposition 6.1: A degree $d_{1}$ Type 2 sequence is reduced to $d_{2}$ by the following operation:

$$
\{\operatorname{Red} \mid \overbrace{\{\mathbb{S}\}}^{d_{1} \text { to } d_{2}}\}=\{\mathbb{S}\} \oplus\left\{\text { uा }_{d=d_{1}-d_{2}}\right\}
$$

The problem of reducement and conducement by AC through Proposition 6.1 is illustrated below by using Example 6.1 in which the parent sequence is reduced to a base sequence. A tabular form is used to reflect on generic changes. Is given in Table 9(a).

Column 2 of Table 8 (a) summarises the reducement operations by symbols with over-fixes, e.g. $3 / 2 / \sigma=1$ (Table $8(\mathrm{a}), \mathrm{ID}=1$ ). This means that the reducement is from hierarchy (degree) 3 into 2 and the sequence source is active at Ordinal 1 (Term 1). The problem of reducing from one hierarchical level to its lower one is through using generic operators with no need for a route map on the architecture of the sequence. The kernel divides the whole space of the hierarchies into the conjugate active and passive spaces. Table 8(a) shows that kernls are produced by reducement from Degree 0 to its next lower level, which is not a sequence but a set of highly ordered numbers and cleansed of the effects of rules.

Remark 6.1: The number of descension steps from the parent sequence to the kernel is as follows: $d=(3-2)+(2-1)+(1-0)+(0-$ kernel $)=4$, as if the provision for priming (transformation of a kernel into a sequence) is counted as one step.

Remark 6.2: Descension from the kernel to the lower hierarchy $(d=-1)$ is direct and no zero level is encountered.

Remark 6.3: A sequence reducing to a kernel is a conducemental sequence. 


\subsubsection{Problem of Conducement from one Hierarchy to Another}

Proposition 6.2: Degree $d_{1}$ Type 2 sequences are conduced from to $d_{2}$ as follows:

$$
\{\text { Cond } \mid \overbrace{\{\mathbb{S}\}}^{d_{1} \text { to } d_{2}}\}=\{\mathbb{S}\} \oplus\{\mathbb{U}\}
$$

The problem of conducement by AC through Proposition 6.2 is illustrated below by using Example 6.1, in which a base sequence is conduced towards the parent sequence. Table 8 (b) presents the conducemental operations to reflect on

Table 8. (a) Problem of reducement: Example 6.1- U1. $\mathbb{U} 1 . \mathbb{U} 1$; (b) problem of conducement: Example 6.1— U1.U1.U1.

(a)

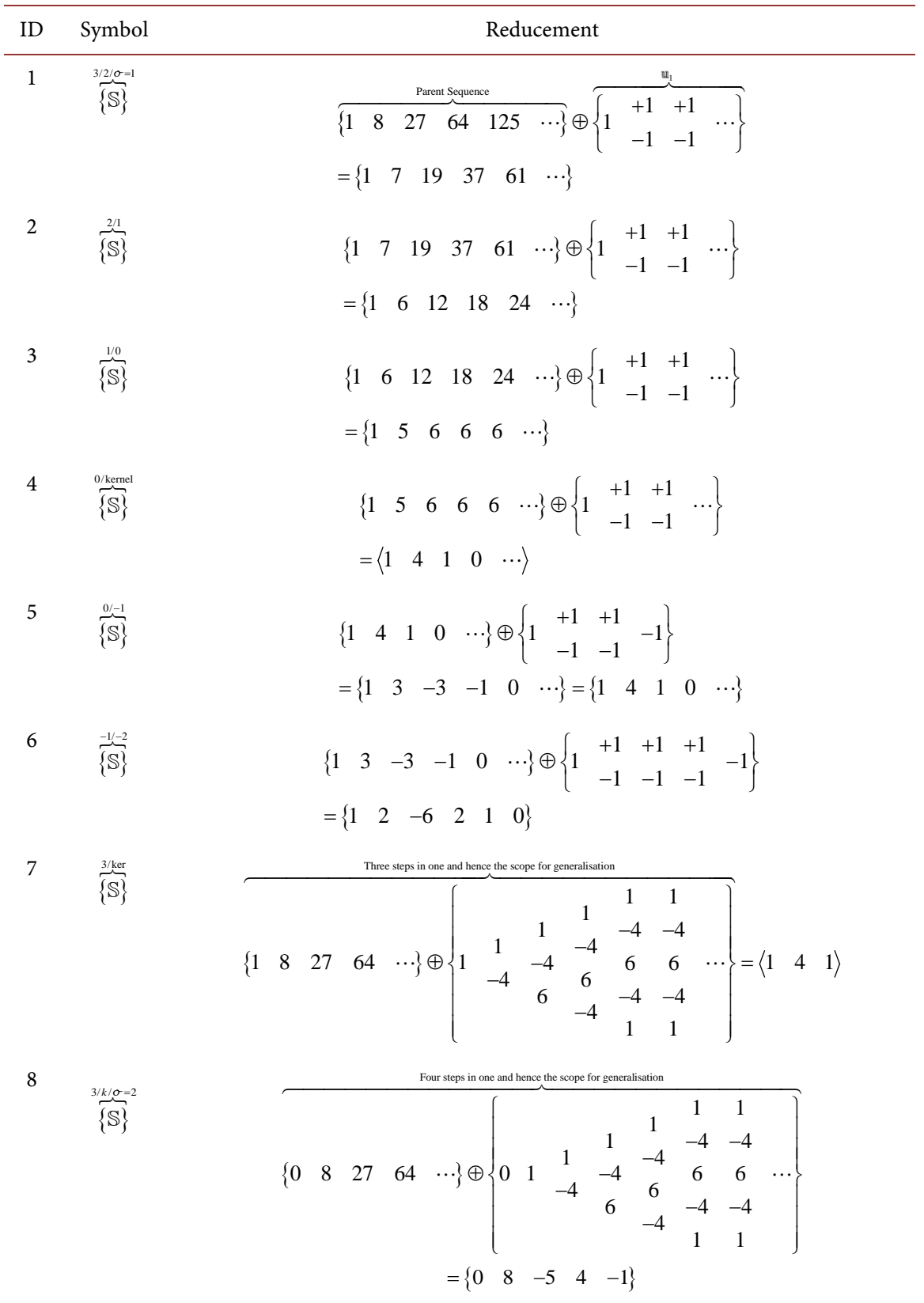


(b)

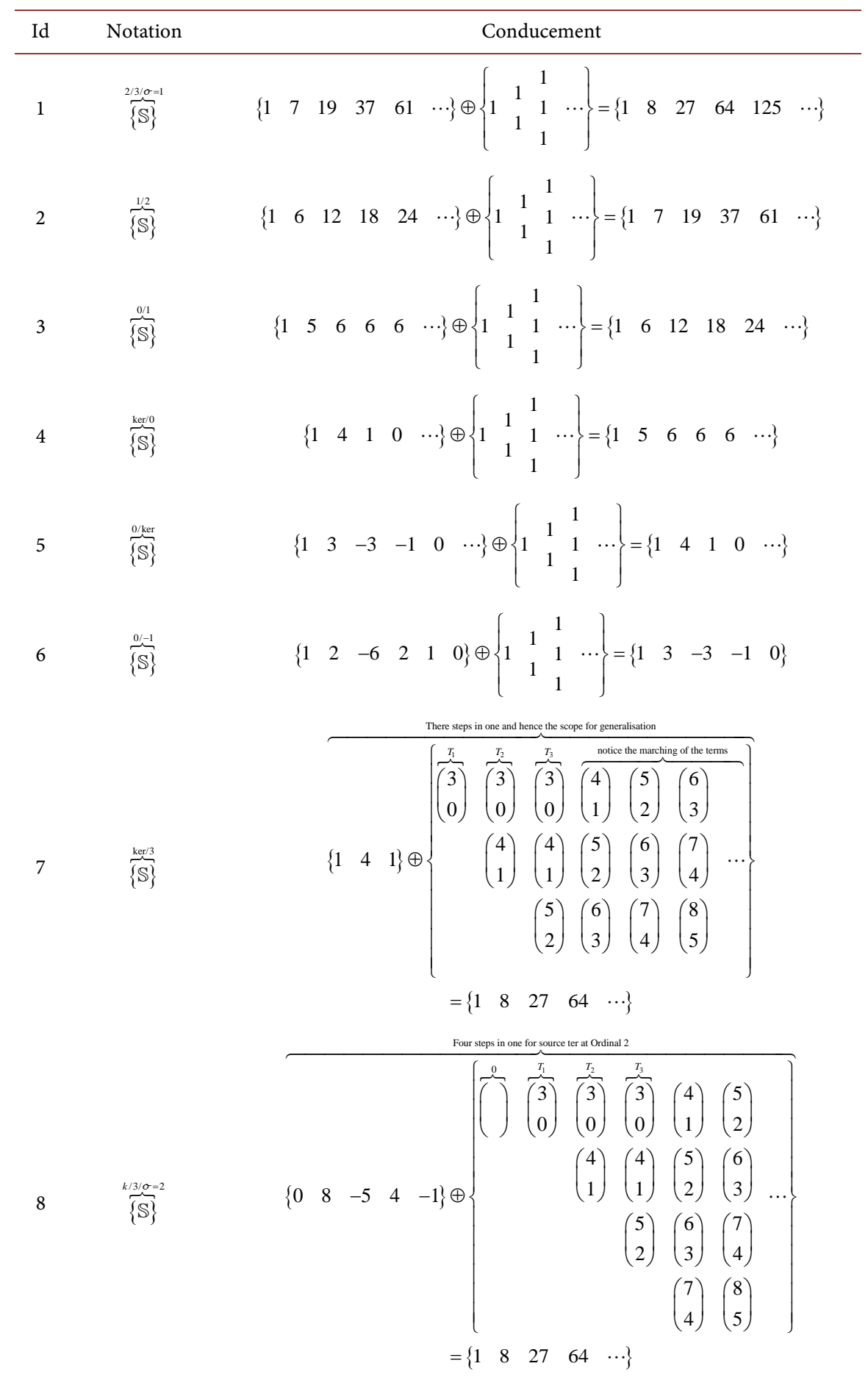

generic changes. Column 2 of Table 8(b) summarises the conducement operations and shows over-fix $2 / 3 / \sigma=1$ at ID $=1$. This is red as: conducement from hierarchy (degree) 2 to 3 with the source term at Ordinal 1.

Remark 6.4: The step for ascension from the kernel to $d=0$ facilitates an additional step. Table 8 (b) at ID 7 shows a few steps are taken at once for conducement opertions. 
Remark 6.5: Conducement and reducement are technical identical operations.

Remark 6.6: Notice the elegance of the replication beyond term 3 at ID 7, as if the gear system of AC is generically adjusted to navigate all the term. To recall the importance of the conducemental networks, parent sequence can start from any source term and the process still is the same.

\subsection{Gradient Operations: Hierarchy to Allele and Vice-Versa}

The paper devises a peculiar numbering system for alleles and this is clearly shown in Table 3 and Table 5. This numbering system plays a pivotal role in the conducemental networks. Whilst the paper uses $\mathcal{A}$ (upper case alpha) to denote alleles, its lower case, $\alpha$, denotes the allele number, which varies from $-\infty$ to $\infty$. The peculiarity of $\alpha$ is that whilst it changes from $-\infty$ to zero (i.e. source terms are active from inner ordinals), the values of degree for each conducemental sequence remains that of the parent sequence. All the alleles from $-\infty$ to zero have the same order.

It is too much of complexity to detail full architectural variations on the alleles between $-\infty$ and $\infty$ in one paper but it has to be stated that $\mathcal{A}_{0}$ is one of transition alleles, $\mathcal{A}_{-1}$ denotes the sequence with its source term being at Ordinal 2 and $\mathcal{A}_{-2}$ at Ordinal 3 and so on. The values of the terms of the sequence prior to the source term are set to zero.

The alleles between $\alpha=0$ and $\alpha=\delta+1$ undergo transitional changes, either their order shrinks or stay constant. These changes are determinate and not accidental or arbitrary. The emergence of the kernel at $\alpha=\delta+1$ should not be seen as a temporary derailment of conducemental algebra but an elegant provision for disappearance of the diagonal and the emergence of a new architecture, in which diagonals (alleles) and sequences are unified below the kernel at the onset pf the passive space.

\subsubsection{Reducement to the Kernel}

The kernel is similar to alleles and both reducement of parent sequences to the kernel and conducement of the kernel to the parent sequence are parallel to the operations given above as per Propositions 6.1 and 6.2.

\subsubsection{Reducement of a Hierarchy to an Allele}

AC operations involving alleles require an architectural route map but the paper is focussed on the simpler Type 2 sequences when $\delta=\omega$ using Example 6.1

Proposition 6.3: Alleles of any parent sequence of degree $d$ or its hierarchy at any degree, say, $d$, are reducible to any of its alleles, $\mathcal{A}_{a}$, by replicators, which need to be "cut-and-shaped" as per architectural route map of the parent sequence. For sequences with $\delta=\omega$, the following rule:

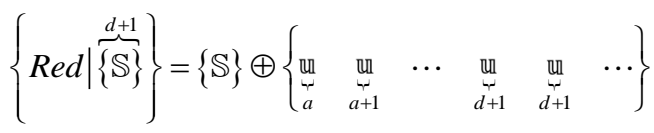


where, $a$ is allele number, $\stackrel{\mathbb{a}}{a}$, is the inverse replicator. The problem of reducing a parent sequence or its hierarchies to any of its allele is evidently dependent on (i) selecting the particular allele $\mathcal{A}_{a}$ or $a$; (ii) both $\delta, \omega$ of the parent sequence; (iii) the type of unbounded conducemental sequences; and (iv) the ordinal of the source term. The problem of reducement from a hierarchy to an allele by AC through Proposition 6.3 is illustrated below by using Example 6.1. Proposition 6.3 is illustrated in a tabular format in Table 9.

Table 9. Reducement of the parent sequence (Example 6.1) to alleles.

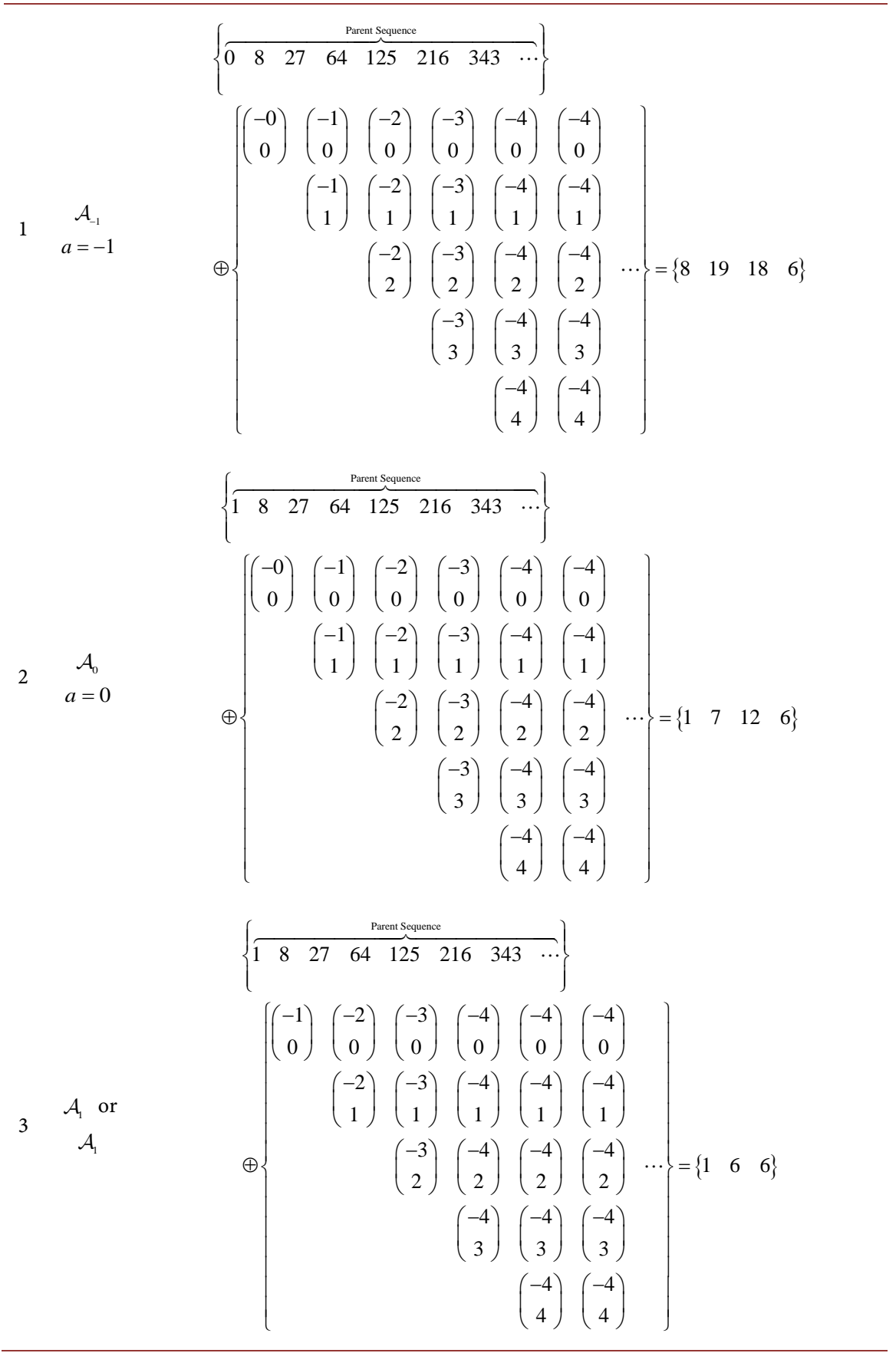




\section{Continued}

${ }_{4} \quad \mathcal{A}_{2}$ or

$\left\{\begin{array}{llllll}1 & 8 & 27 & 64 & 125 & \cdots\end{array}\right\}$

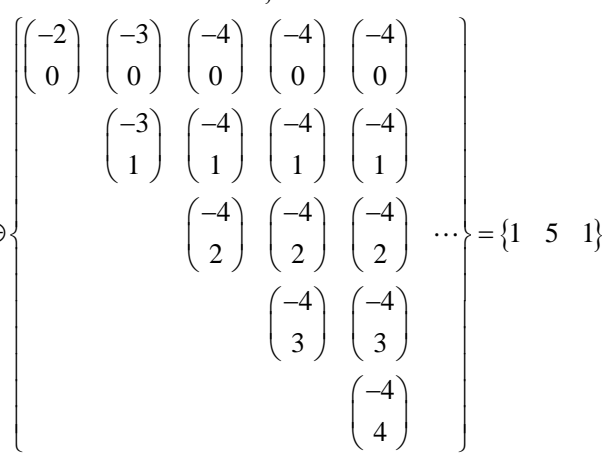

$\left\{\begin{array}{llllll}1 & 8 & 27 & 64 & 125 & \cdots\end{array}\right\}$

${ }_{5} \quad \mathcal{A}_{3}$ or

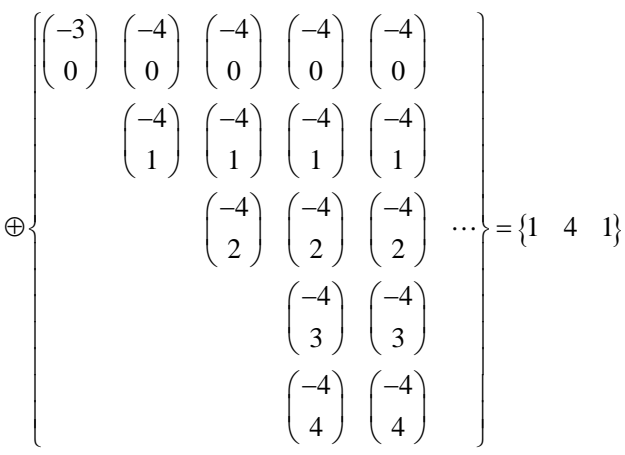

$\left\{\begin{array}{llllllll}1 & 8 & 27 & 64 & 125 & 216 & 343 & \cdots\end{array}\right\}$

$6 \quad$ Ker

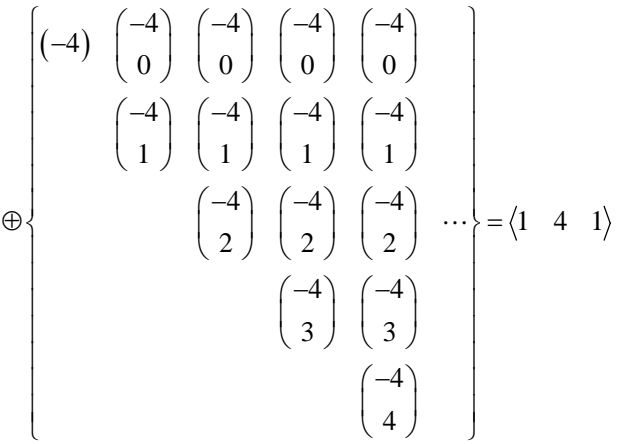

$\left\{\begin{array}{llllllll}1 & 8 & 27 & 64 & 125 & 216 & 343 & \cdots\end{array}\right\}$

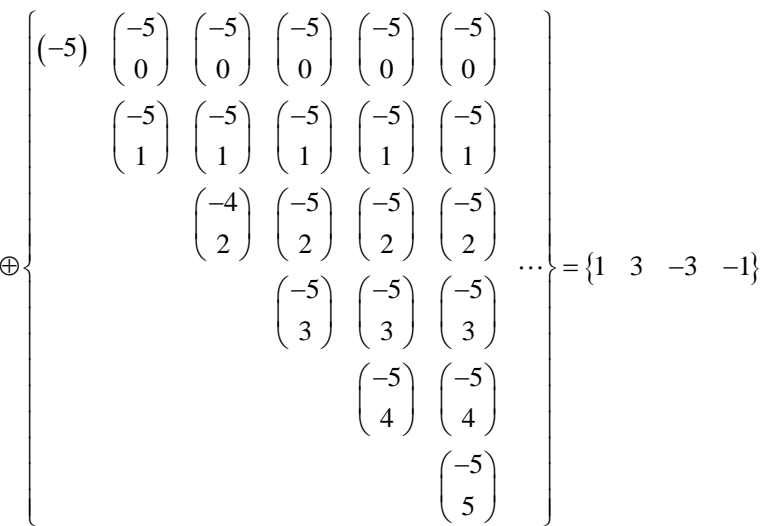


Remark 6.7: The under-fix in $\stackrel{\square}{a}$ is a shorthand way for representing the regeneration operation.

\subsubsection{Conducement of an Allele to a Hierarchy}

AC operations for generating a parent sequence or its hierarchies from its alleles require an architectural route map. The proposition below expresses the problem for Type 2 sequences.

Proposition 6.4: Alleles can be conduced to its parent sequence of degree $d$ or of its hierarchies at any specified degree by replicators but replicators need to be "cut-and-shaped" as per architectural route map. For sequences with $\delta=\omega$, the following rule:

$$
\left\{\operatorname{Red} \mid\{\stackrel{d+1}{\mathbb{S}\}}\}=\{\mathbb{S}\} \odot\left\{\mathcal{U}_{a}\right\}\right.
$$

where, $\mathcal{U}_{a}$ is the replicator. The problem of conducing an allele to a parent sequence or its hierarchies is dependent on (i) both $\delta, \omega$ of the parent sequence; (ii) the type of unbounded conducemental sequences; (iii) the ordinal of the source term. The problem of conducement from an allele to a hierarchy by AC through Proposition 6.5 is illustrated, in Table 10.

Remark 6.8: These investigations use an alternative formulation to be presented in the near future in more detail including the allele $\alpha=0$ and a set of other transition alleles.

Table 10. Conducing from alleles to parent sequences.

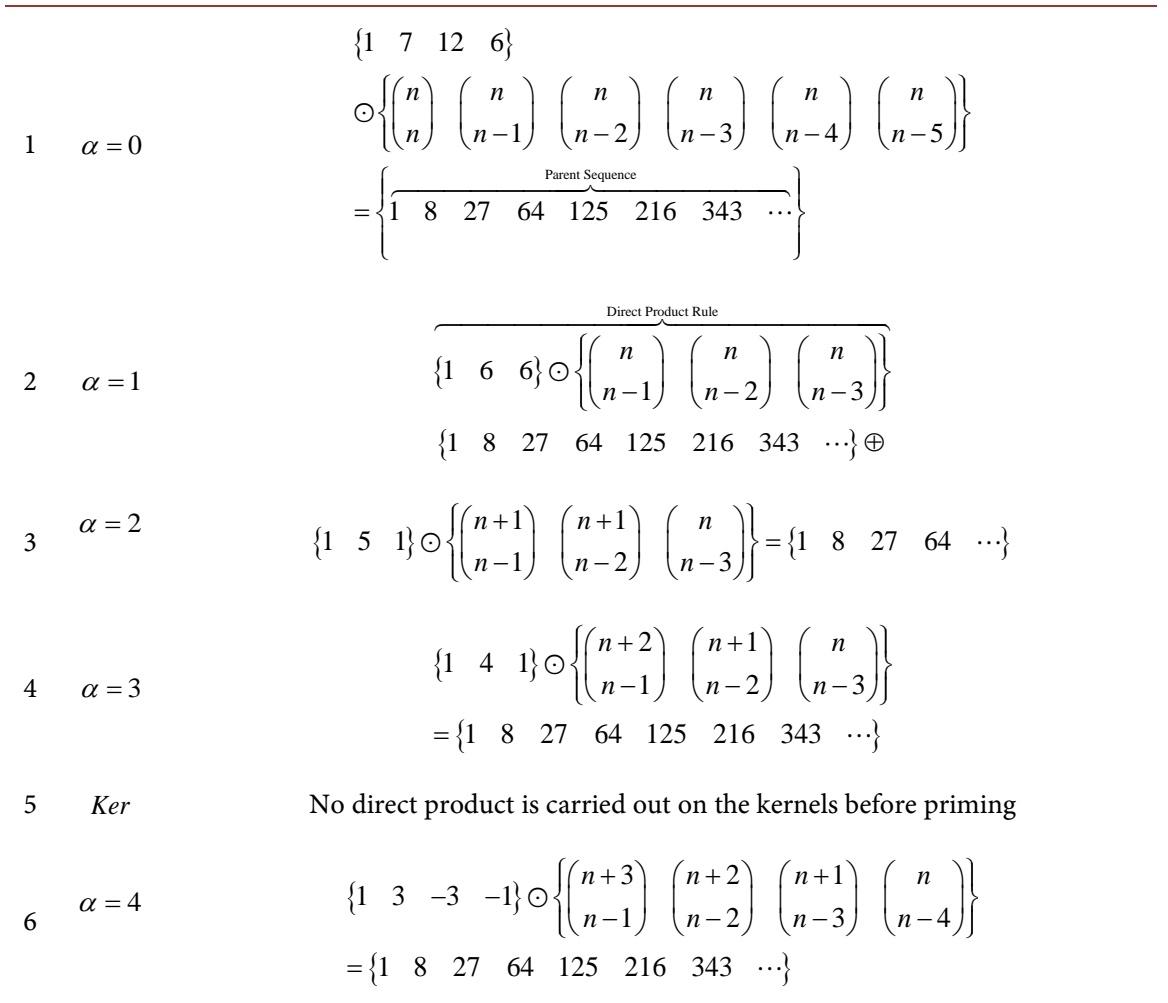




\subsection{Operations on Allele-to-Allele}

\subsubsection{Reducement from One Allele to Another}

Table 11 exemplifies reducements of alleles to alleles using Example 6.1 without presenting any proposition, as the paper is mainly focused on initiating background for AC.

As may be seen from Table 11, AC provides tools to operate on one allele to obtain the values of another allele, which also depends on the architecture of the sequence, on the ordinal of the source term and the type of the sequence.

\subsubsection{Conducement from One Allele to Another}

The conducement of one allele into another allele is not also presented in any depth, as a deeper understanding of the architecture isrequired and therefore its introduction suffices to tabulating the above sequences in Table 12.

It is noted that the replicators for both reducements and conducement in $\mathrm{Ta}$ ble 10 to Table 11 operations are handled by the binomial bounded conducemental sequences $\mathcal{U}$ and their inverse $u$, as alleles are bounded sequences.

Table 11. Reducement of one allele to another.

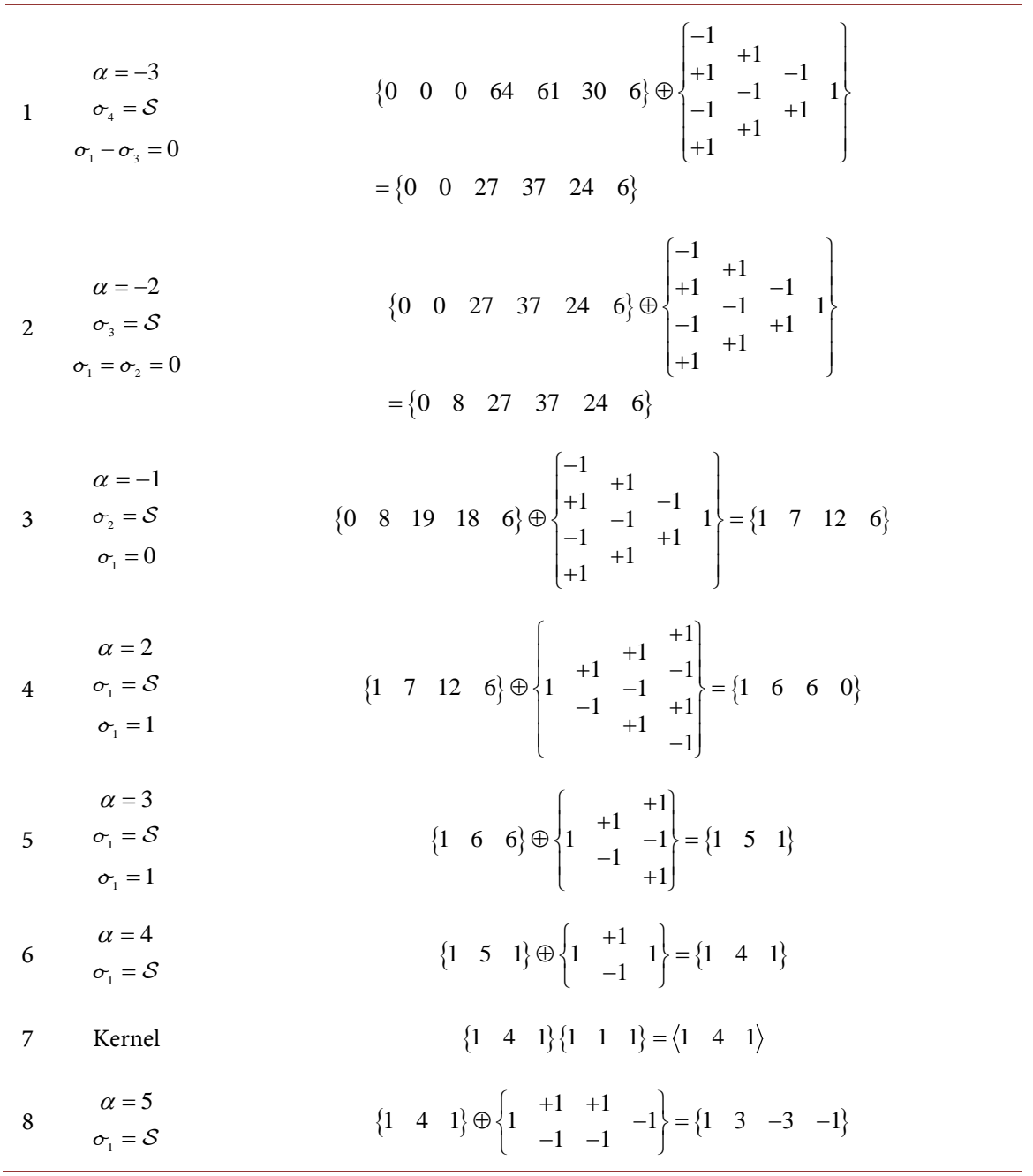


Table 12. Conducement from one allele to another.

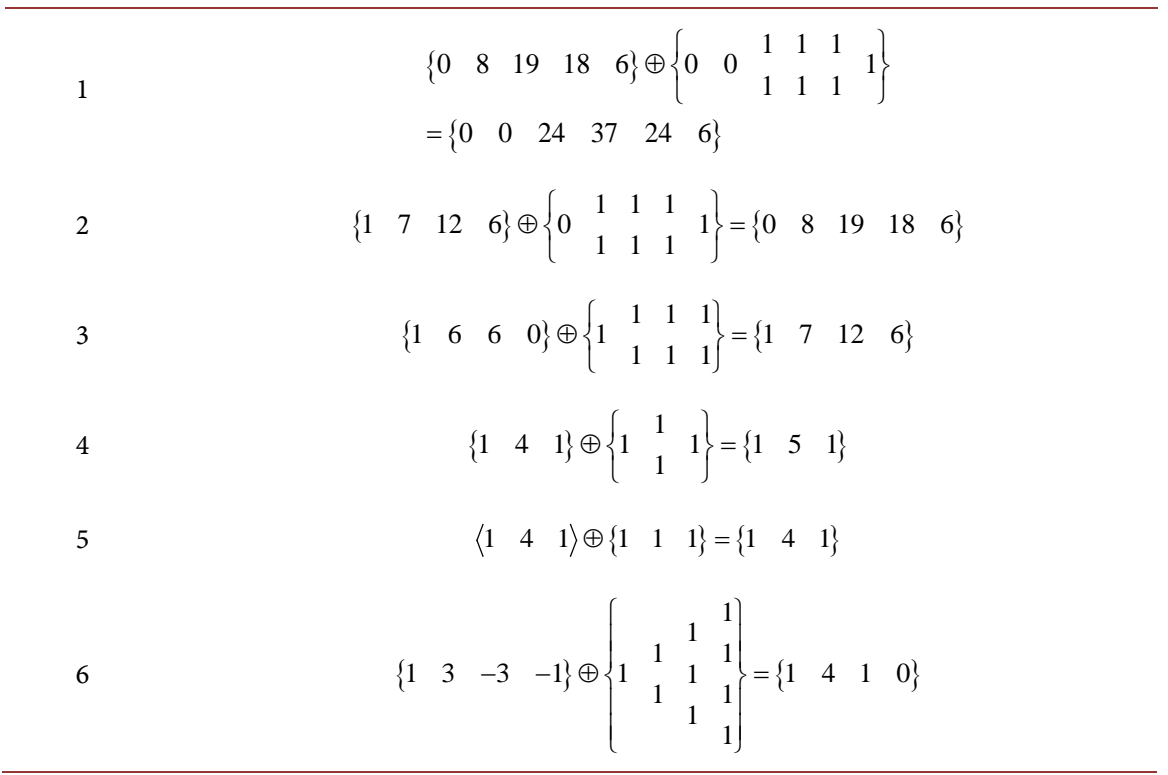

\subsection{Interoperability Operations}

Interoperability is defined as the capability to operate more than one step at a time, where the steps are measured in terms of one degree or generation. It is most elegant part of AC and provides tools to overcome the fear of the new conducemental network, without which the network appears as a maze with a high risk of getting lost within or being entangled somewhere in it. This subject is not also presented in any specific detail but in terms of using Example 6.1 and related to the interoperability among alleles (allele to allele). The idea is presented in Table 13, which shows the cogwheels of AC are not fixed but highly versatile by taking more than one step (one step is one degree) at a time.

The author has developed algorithms to express interoperability from one allele to another, from one hierarchy to an allele from one hierarchy to another but the length of the paper does not allow any greater presentation.

\subsection{Operations on Structure of Hierarchies}

Section 5 of the paper provides examples to show the way the uninomial sequences can be used to display the structure of hierarchies and to reflect on internal structures. This now used as a model to display the structure of Example 6.1 for one of the term.

Example 6.1 is expressed as:

$$
\left\{\begin{array}{lllll}
\text { Kernel } \mid 1^{3} & 2^{3} & 3^{3} & \cdots & n^{3}
\end{array}\right\}\left\langle\begin{array}{lll}
1 & 4 & 1 \\
i & 2 & 3
\end{array}\right\}
$$

This is a Degree 3 sequence and therefore Degree 3 uninomial sequence, $\mathbb{U}_{3}$, is used as a model to display the structure of hierarchies from Term 1 to Term 4, as expressed by (5.5c), in which the terns, sub-terms and sub-sub-terms are filled by the kernel values: 
Table 13. Illustrating interoperability by example.

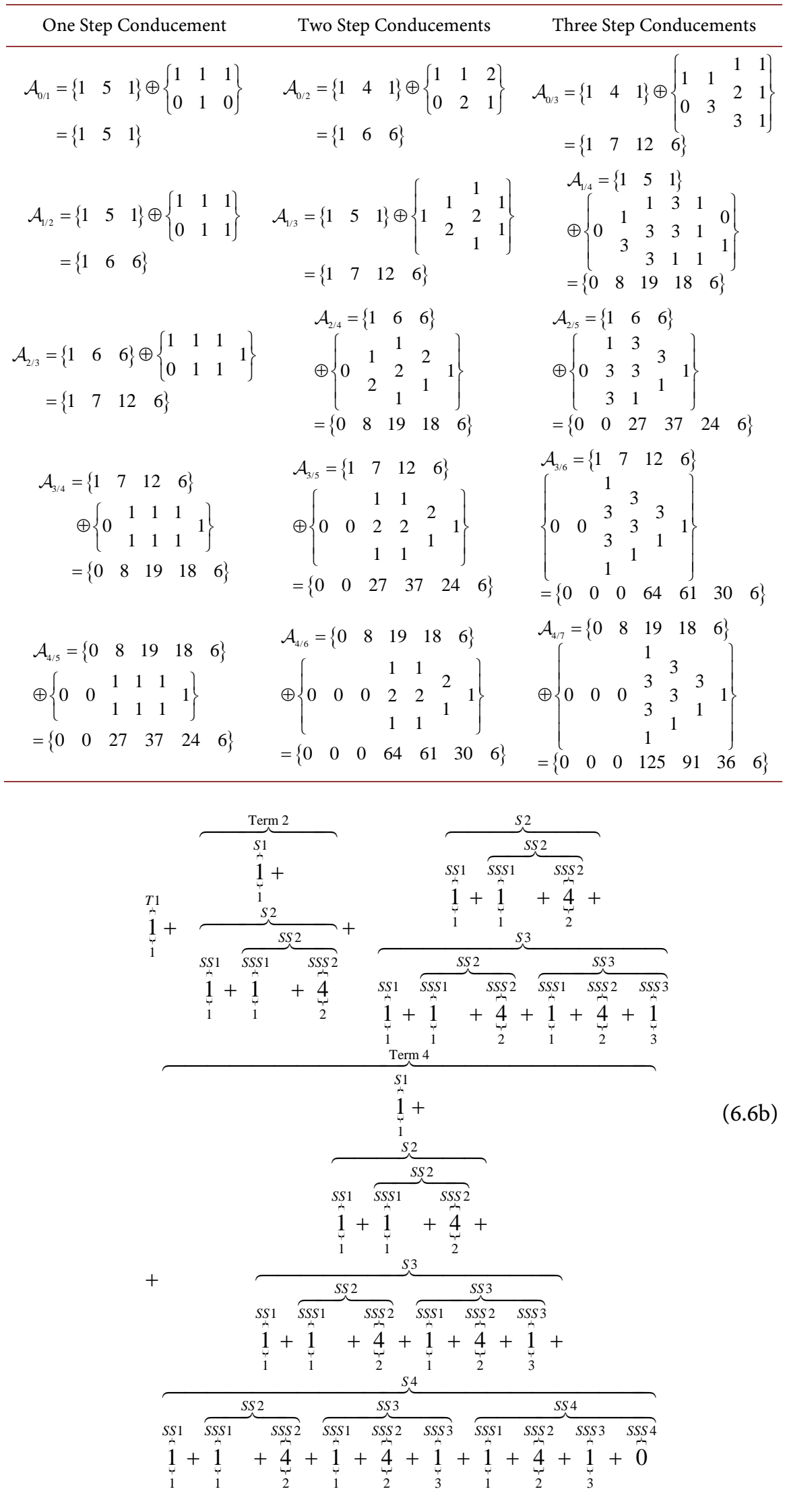




\section{Discussion}

Arithmetic Calculus (AC) has the potential for a game change in mathematics but the price for it is the consensus to its uptake, change of habit, the acquisition of a new discourse. These are not automatic processes but the paper makes the case by showing that even most basic operations, e.g. $2 \times 3$, are embedded with AC. Literally any single mathematical operation requiring reducement is the deconvolution problem but AC evidently copes with these operations analytically. Perhaps this should be enough incentive to critically question this widelyknow deconvolution theorems. However, old habits die hard even in mathematics.

Attention is drawn to the conducemental network towards the big picture on the architectural variations in Type 2 to unbounded conducemental sequences. This is necessary to cut-and-shape replicators based on a route map using conducemental network. The network is the difference table but with a new lease of life, as it is transformed into a grid-like network using degree, ordinal and diagonals. There is one route map for each type of conducemental sequences reflecting architectural variations, as discussed in Sections 5 and 6 together with Table 3 and Table 5.

Architecture is used in the paper to refer to generic variations in the operation from one allele to another, which depends on degree and order and is complemented by contributions from the logistics of the parent sequence. There is one architecture for each type of the conducemental sequences. Whilst a full account of architectural variations will be presented after this paper, Example 6.1 provides a glimpse of architecture for Type 2 sequences. This is generalised for the cases where degree $\geq$ order and shown in Figure 1. Similar architectures have been studied by the author for Types 3 and 4 unbounded conducemental sequences but are not presented here due to the scope of the paper.

It is not within the remit of this paper to catalogue the shortfalls of classic mathematics but consider the concealment concept using $\mathbb{T}_{2}$ (see Table 1, ID 10), as follows:

$$
\begin{aligned}
& \left\{\mathbb{T}_{2}\right\}=\left\{\begin{array}{lllllll}
1 & 4 & 10 & 18 & 27 & 36 & \cdots
\end{array}\right\} \\
& =\left\{\begin{array}{lllll}
1 & 2 & 3 & 2 & 1
\end{array}\right\} \odot\left\{\left(\begin{array}{c}
n \\
n-1
\end{array}\right)\left(\begin{array}{l}
n-1 \\
n-2
\end{array}\right)\left(\begin{array}{l}
n-2 \\
n-3
\end{array}\right)\left(\begin{array}{l}
n-3 \\
n-4
\end{array}\right)\left(\begin{array}{l}
n-4 \\
n-5
\end{array}\right)\right\}
\end{aligned}
$$

This is expressed algebraically, as follow:

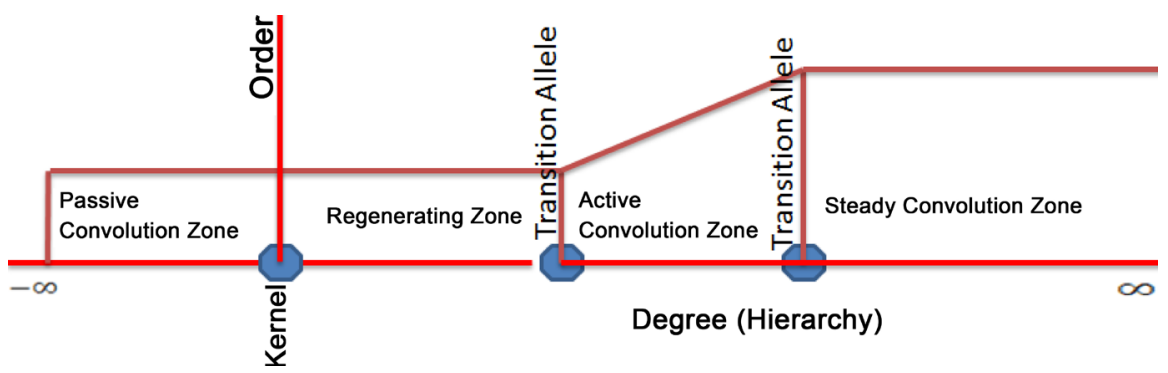

Figure 1. Architecture of orders and allele-Types 2 unbounded sequences. 


$$
1(n)+2(n-1)+3(n-2)+2(n-3)+1(n-4)=9 n-18
$$

(7.1) is an AC expression, which is correct for any value of $n$ but (7.2) is its equivalent version in classic algebra, which fails at $n=1,2$ and 3 (this may be called as a singularity problem, or rather the triple singularity problem). Besides, $\mathrm{AC}$ reflects on the architecture and the structure of the sequence but algebra has no such senses.

Attention is drawn to the fact that the examples presented in the paper have at least 100 instances of the inverse problem (the deconvolution problem). Evidently, AC has no problems in treating the deconvolution problem.

\section{Conclusions}

The paper has the primary aims of advancing the status of AC from Technical Readiness Level of 1 to 2 and naturally the presentation is not yet rigorous, although the author has developed a rigorous proof for the fundamental premises of AC. The paper contextualises AC in terms of transforming the age-old difference tables into a new network, referred to as the conducemental network, reminiscent of other coordinate systems, such as the Cartesian system. The network is bounded between the parent sequence and its base sequence, which are selected for convenience. Its rows are referred to as hierarchies and each hierarchical level is identified by its degree; whereas the columns are identified by ordinal numbers. The source of a sequence at a hierarchical level can be activated from any ordinal number and this sets diagonals, which retain the full knowledge about the sequence, like "stem cells" in biology. The paper presented a glimpse of the architecture of AC sequences but the full presentation of this subject is outside the remit of the paper.

The paper categorised conducemental sequences into five types, which collectively can cope with any type of polynomial and exponential functions. The focus of the paper is on Types 1 and 2 sequences but another paper has been prepared to cover Type 3 and 4 and to complete the picture on the architectural variety of conducemental sequences. Yet another paper is under preparation to cover Type 5 sequences.

The paper also shows a glimpse of the big picture on the architectural variations in Type 2 unbounded conducemental sequences, the understanding of which is necessary to cut-and-shape replicators to facilitate the following operations: (i) reducement and conducement from one hierarchy to another; (ii) reducement and conducement between hierarchies and allele; (iii) reducement and conducement between alleles; (iv) interoperability; and (v) reconstruction of the structure of each term. Replicators are the cogwheels of AC and facilitate complex operations in explicit terms.

\section{Acknowledgements}

The paper is the outcome of 22 years of the author's arduous scholarly research on AC. Moral consent is only given to open science and open mathematics ap- 
plications of AC and its derivatives but no moral consent to closed coding or use. Science and mathematics need an open environment to thrive and be in the service of all.

\section{References}

[1] Khatibi, R. (2014) Introducing “Arithmetic Calculus" with Some Applications: New Terms, Definitions, Notations and Operators. Applied Mathematics, 5, 2909-2934. http://www.scirp.org/journal/PaperInformation.aspx?PaperID=51184

[2] NASA's Technology Readiness Level (TRL).

https://www.nasa.gov/directorates/heo/scan/engineering/technology/txt_accordion 1.html https://www.nasa.gov/sites/default/files/trl.png

[3] Dominguez-Torres, Alejandro (2010) Origin and history of convolution. $41 \mathrm{p}$. http://www.slideshare.net/Alexdfar/origin-adn-history-of-convolution

[4] Wilf, H.S. (1994) Generating Functionology. http://www.math.upenn.edu/ wilf/gfology2.pdf

[5] Qu, A.J. (1997) Revisiting an Eighth-Century Chinese Table of Tangents, Chapter 3, History of Oriental Astronomy: Proceedings of the Joint Discussion-17 at the 23rd General Assembly of the International Astronomical Union, Organised by the Commission 41 (History of Astronomy), Held in Kyoto, 25-26 August, 1997; Editors: Ansari, S.M. (Ed.) 


\section{Appendix I. Reformulation of the Convolution Theorem}

The mathematical community does not have the prior knowledge of AC; whereas initiation for algebraic calculus takes place from secondary school. Thus, AC starts without the privilege of initiation but this section anchors AC on firmly established mathematical theorems.

AC can reveal so much about mathematics but to get to that stage, many mundane mathematical operations have to be approached differently. Whilst it is recognised that old habits die hard, this section shows that new habits are beneficial and when these benefits are realised, insistence on old habits are likely to be seen as insistence on carrying on with Roman numerals compared with Arabic-Indian numerals. The paper relies on the mathematical flair of the readership, who are prepared to resort to the good old habit of working out mathematics using paper and pen, without which, the benefit from AC can be very little.

Application of the Convolution Theorem to Hydrology: One of the known applications of the convolution theorem in its discrete form is the unit hydrograph theorem in hydrology.

According to the theorem, let $\boldsymbol{R}=\left\{\begin{array}{llll}R_{1} & R_{2} & \cdots & R_{m}\end{array}\right\}$ be the array of effective rainfall $U=\left\{\begin{array}{llllll}U_{1} & U_{2} & \cdots & U_{m} & \cdots & U_{n}\end{array}\right\}$ be unit hydrograph ordinates $\boldsymbol{Q}=\left\{\begin{array}{llllllll}Q_{1} & Q_{2} & \cdots & Q_{m} & \cdots & Q_{n} & \cdots & Q_{m+n-1}\end{array}\right\}$ be surface water runoff

The unit hydrograph theorem is often expressed as:

$$
\boldsymbol{R} * U=\boldsymbol{Q}_{(\mathrm{I} .0)}
$$

which is expanded as:

$$
\begin{gathered}
R_{1} * U_{1}=Q_{1} \\
R_{1} * U_{2}+R_{2} * U_{1}=Q_{2} \\
R_{1} * U_{3}+R_{2} * U_{2}+R_{3} * U_{1}=Q_{3} \\
R_{1} * U_{m}+R_{2} * U_{m-1}+\cdots+R_{m-1} * U_{2}+R_{m} * U_{1}=Q_{m} \\
R_{1} * U_{m+1}+R_{2} * U_{m}+\cdots+R_{m-1} * U_{3}+R_{m} * U_{2}=Q_{m+1} \\
\cdots \\
R_{1} * U_{n}+R_{2} * U_{n-1}+\cdots+R_{m-1} * U_{n-m+2}+R_{m} * U_{n-m+1}=Q_{n} \\
R_{2} * U_{n}+\cdots+R_{m-1} * U_{n-m+3}+R_{m} * U_{n-m+2}=Q_{n+1}( \\
\cdots \\
R_{m} * U_{n}=Q_{n+m-1}
\end{gathered}
$$

The above is true for rainfall-runoff models and equally true in AC. The only shorthand representation for (I.1) is (I.0) but the alternative of AC transformation is as follows:

$$
\begin{aligned}
& \left\{\begin{array}{llll}
R_{1} & R_{2} & \cdots & R_{m}
\end{array}\right\} \hat{\oplus}\left\{\begin{array}{llllll}
U_{1} & U_{2} & \cdots & U_{m} & \cdots & U_{n}
\end{array}\right\} \\
& =\left\{\begin{array}{llllllll}
Q_{1} & Q_{2} & \cdots & Q_{m} & \cdots & Q_{n} & \cdots & Q_{m+n-1}
\end{array}\right\} \\
& =\left\{\begin{array}{llllllll}
(\text { I.1a) } & \text { (I.1b) } & \cdots & \text { (I.1d) } & \cdots & \text { (I.1f) } & \cdots & \text { (I.1h) }
\end{array}\right\}
\end{aligned}
$$


which may be written?

$$
\begin{aligned}
& \left\{\begin{array}{ccccc} 
& & & R_{1} \\
R_{1} & R_{1} & R_{1} & & R_{2} \\
& R_{2} & R_{2} & \cdots & R_{3} \\
& R_{3} & & \vdots \\
& & & & R_{m}
\end{array}\right\} \hat{\oplus}\left\{\begin{array}{ccccccc} 
& & & & U_{1} & & \\
& & U_{2} \\
& U_{1} & & & U_{2} & & U_{3} \\
U_{1} & U_{2} & \cdots & U_{3} & \cdots & \vdots \\
& & U_{3} & & \vdots & & U_{m} \\
& & & & U_{m} & & \vdots \\
& & & & & & U_{n}
\end{array}\right\}
\end{aligned}
$$

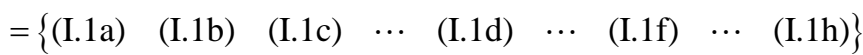

The symbol $\hat{\oplus}$ signifies a new kind of operations, in which the multiplications are carried out in a very peculiar way. For instance, the operations on the third term, i.e. (ESM1.1c), are illustrated below:

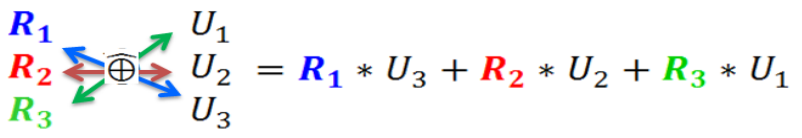

The operations (I.0)-(I.1) describe traditional form of the convolution theorem for discrete variables. AC expresses these operations in a sequence form as laid out by (I.2), and the peculiar way of multiplying the terms of $\{R\}$ and $\{U\}$ triggered by the symbol $\hat{\oplus}$ cannot be overemphasised. The medium of a mathematical paper is necessarily limited in its power of communication for the uninitiated readership and the only compensation for this is mathematical flair. Notably, there is nothing to be proved so far, as the convolution theorem is well established.

An alternative form of (I.2a)-(I.2b) is to fill in the remaining terms of $\{R\}$ and $\{U\}$ with zero and make them size of $m+n-1$ and change the multiplication from convolution to regeneration product, as follows:

$$
\begin{aligned}
& \left\{\begin{array}{llllllllll}
R_{1} & R_{2} & \cdots & R_{m} & 0_{m+1} & \cdots & 0_{n} & 0_{n+1} & \cdots & 0_{n+m-1}
\end{array}\right\} \\
& \oplus\left\{\begin{array}{lllllllll}
U_{1} & U_{2} & \cdots & U_{m} & \cdots & U_{n} & 0_{n+1} & \cdots & 0_{n+m-1}
\end{array}\right\}
\end{aligned}
$$

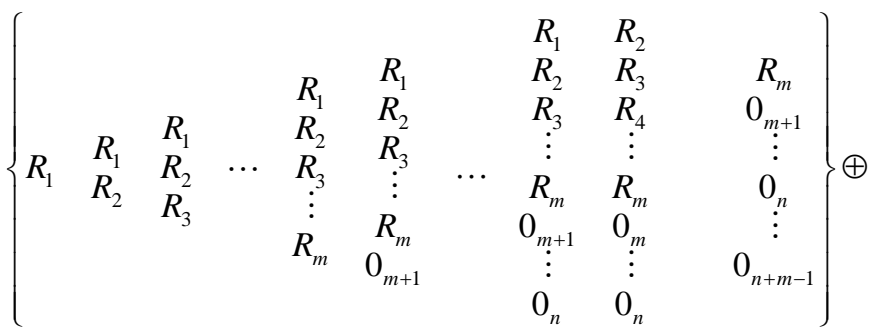

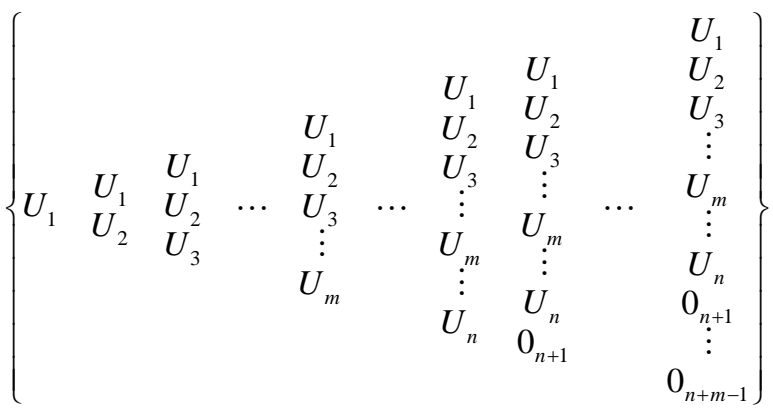




$$
\begin{aligned}
& \left\{\begin{array}{lllllllll}
(\text { I.1a) } & \text { (I.1b) } & \text { (I.1c) } & \cdots & \text { (I.1d) } & \cdots & \text { (I.1f) } & \cdots & \text { (I.1h })
\end{array}\right\} \\
& =\left\{\begin{array}{llllllll}
Q_{1} & Q_{2} & \cdots & Q_{m} & \cdots & Q_{n} & \cdots & Q_{m+n-1}
\end{array}\right\}
\end{aligned}
$$

Readers should confirm to themselves that the above is true by carrying out the operations. Besides, (I.1) is taken to be improperly posed, as the number of equations $(n+m-1)$ is greater than the unknowns, which are as many as $(n)$ or $(\mathrm{m})$. However, this is not true and AC will show that the reducement problem is the inverse problem amenable to exact treatments, although it can suffer from ill-conditioning. 